\title{
L'2-SIGNATURES, HOMOLOGY LOCALIZATION, AND AMENABLE GROUPS
}

\author{
JAE CHOON CHA AND KENT E. ORR
}

\begin{abstract}
Aimed at geometric applications, we prove the homology cobordism invariance of the $L^{2}$-betti numbers and $L^{2}$-signature defects associated to the class of amenable groups lying in Strebel's class $D(R)$, which includes some interesting infinite/finite non-torsion-free groups. The proofs include the only prior known condition, that $\Gamma$ is a poly-torsion-free abelian group (or potentially a finite $p$-group.) We define a new commutator-type series which refines Harvey's torsion-free derived series of groups, using the localizations of groups and rings of Bousfield, Vogel, and Cohn. The series, called the local derived series, has versions for homology with arbitrary coefficients, and satisfies functoriality and an injectivity theorem. We combine these two new tools to give some applications to distinct homology cobordism types within the same simple homotopy type in higher dimensions, to concordance of knots in three manifolds, and to spherical space forms in dimension three.
\end{abstract}

\section{INTRODUCTION}

In their paper 14, Cochran, Orr, and Teichner introduced $L^{2}$-signature defects (equivalently, von Neumann $\rho$-invariants, or Cheeger-Gromov invariants) to study concordance of knots, or more generally, homology cobordism classes of 3-manifolds. They showed the invariance of $L^{2}$-signature defects under integral homology cobordism over a poly-torsion-free abelian group, that is, a group with a descending series admitting successive $\mathbb{Z}$-torsion free quotients. Since then, $L^{2}$-signatures have appeared as a key ingredient of several interesting papers on homology cobordism and concordance by authors including Cha, Cochran, Friedl, Harvey, Heck, Horn, Kim, Leidy, Orr, and Teichner.

This paper substantially extends the above $L^{2}$-invariance under homology cobordism to a much larger class of groups, and incorporates homology with twisted coefficients as well.

Achieving this result requires a small shift of paradigm. The Cochran-OrrTeichner results used the following property of a poly-torsion-free abelian group $\Gamma$ : the group ring $\mathbb{Z} \Gamma$ embeds into a skew field which is a module over the Cohn localization of $\mathbb{Z} \Gamma$. This implies that a homology cobordism looks like a product to the skew field. For the groups $\Gamma$ we consider, $\mathbb{Z} \Gamma$ may not embed in a skew field, requiring an entirely new approach. Our new approach, which uses neither Cohn localization nor a skew field, subsumes poly-torsion-free abelian groups as a special case. We employ directly $L^{2}$-methods with coefficients in the von Neumann algebra $\mathcal{N} \Gamma$ by using a result of Lück on $L^{2}$-dimension zero modules (see Theorem 6.3).

We consider the class $D(R)$ of groups defined and studied by Strebel 28]; for a commutative ring $R$ with unity, a group $G$ lies in $D(R)$ if given any homomorphism of projective $R G$-modules $\alpha: P \rightarrow Q$, then if $\alpha \otimes 1_{R}$ injective so is $\alpha$. Our result below applies to the class of amenable groups in $D(R)$, a class which includes

2000 Mathematics Subject Classification. 20J05, 57M07.

Key words and phrases. Cohn localization, Bousfield localization, Vogel localization, Derived Series, Injectivity. 
interesting infinite and finite non-torsion-free groups, as well as, using the case $R=\mathbb{Z}$, poly-torsion-free abelian groups (e.g., see Lemma 6.8). The following theorem gives a special case of Theorem 7.1 .

Theorem. Suppose $G$ is an amenable group lying in Strebel's class $D(R)$. If $W$ is an $R$-homology cobordism between two closed $n$-manifolds $M$ and $M^{\prime}$ with restrictions to group homomorphisms $\phi$ and $\phi^{\prime}$ of $\psi: \pi_{1}(W) \rightarrow G$ to $\pi_{1}(M)$ and $\pi_{1}\left(M^{\prime}\right)$, then the $L^{2}$-signature defects $\rho(M, \phi)$ and $\rho\left(M^{\prime}, \phi^{\prime}\right)$ are equal.

To prove this, we use a new technique to control the $L^{2}$-dimension of homology with von Neumann group algebra $\mathcal{N} \Gamma$ coefficients, as shown in the following special case of Theorem 6.6. This not only plays a key role in the proof of the above theorem, but we anticipate using this for future applications as well:

Theorem. Suppose $G$ is an amenable group in $D(R)$, and $C_{*}$ is a finitely generated free chain complex over $\mathbb{Z} \Gamma$. View $R$ as a $\mathbb{Z} \Gamma$-module with trivial $\Gamma$-action. If $H_{*}\left(C_{*} \otimes_{\mathbb{Z} \Gamma} R\right)=0$, then $H_{*}\left(C_{*} \otimes_{\mathbb{Z} \Gamma} \mathcal{N} \Gamma\right)$ has $L^{2}$-dimension zero.

We apply our new theorem on $L^{2}$-signatures to study homology cobordism classes of topological manifolds in dimension three and higher, focusing on space forms, and three manifolds whose groups have torsion elements.

We also employ an additional and essential new tool for these results - a new commutator series, analogous to the Harvey derived series of a group [10, but often much smaller, allowing us to extract additional information from quotients. We prove a type of Stallings injectivity theorem, similar in character to those first developed by Cochran and Harvey in 10, and proved similarly. The significance of this new series over the Harvey series is the use of the Cohn localization of rings and modules, which yields a functorial and often computable series, in place of the Ore localization used by Harvey. To define this series, we use group localization as well. We call this new series, which can be defined for any coefficient $R$, the Vogel-Cohn $R$-local derived series. We define and investigate the analogous series using Bousfield localization as well. (For more details, see Section 1.2 and 3.1.)

As an application involving non-torsion-free groups, we give a homology cobordism version of a theorem of Chang and Weinberger [8] on homeomorphism types of manifolds with a given homotopy type. In this paper we denote by $\mathbb{Z}_{(p)}$ the classical localization of $\mathbb{Z}$ away from $p$, while $\mathbb{Z}_{p}$ denotes $\mathbb{Z} / p \mathbb{Z}$.

Theorem 8.2. Suppose $M$ is a closed $(4 k-1)$-manifold with $\pi=\pi_{1}(M), k \geq 2$. Let $p$ be prime and $\pi^{(n)}$ be the $\mathbb{Z}_{p}$ or $\mathbb{Z}_{(p)}$-coefficient Vogel-Cohn local derived series of $\pi$. If $\pi$ has a torsion element which remains nontrivial in $\pi / \pi^{(n)}$ for some $n$, then there exist infinitely many closed $(4 k-1)$-manifolds $M_{0}=M, M_{1}, M_{2}, \ldots$ such that each $M_{i}$ is simple homotopy equivalent and tangentially equivalent to $M$ but $M_{i}$ and $M_{j}$ are not homology cobordant for any $i \neq j$.

In the proof, we make use of a nonvanishing property for certain $L^{2}$-signatures associated to non-torsion-free groups due to Chang and Weinberger [8], and apply our result to capture the invariance of these $L^{2}$-signatures under homology cobordism as well as homeomorphism. The $\mathbb{Z}_{p}$ coefficient analogue of Theorem 8.2 holds as well (see Section 8.1.)

Additionally we apply these techniques to spherical 3-space forms and prove the following. We say that $M$ is homology equivalent to $N$ if there is a map $M \rightarrow N$ which induces isomorphisms on the homology.

Corollary 8.5. For any generalized quaternionic spherical 3-space form $M$, there are infinitely many closed 3-manifolds $M_{0}=M, M_{1}, M_{2}, \ldots$ such that the $M_{i}$ are homology equivalent to $M$ and have identical Wall multisignatures (or equivalently 
Atiyah-Singer $G$-signatures) and Harvey $L^{2}$-signature invariants $\rho_{n}[19$, but no two of the $M_{i}$ are homology cobordant.

1.1. Injectivity theorems. The surprising injectivity theorem of Cochran and Harvey [10] compares the commutator series quotients of groups with similar homological properties, much as Stallings' famous theorem did for the lower central series. By an injectivity theorem, we mean a theorem of the type first proven by Cochran and Harvey [10, and modeled after Stallings' seminal work on the low dimensional homology of groups and central series [27. Cochran and Harvey work with a suitable commutator series of a group first suggested by Harvey, and which we call here the Harvey series. They show that every group homomorphism $\pi \rightarrow G$ which induces an isomorphism $H_{1}(\pi, \mathbb{Q}) \rightarrow H_{1}(G, \mathbb{Q})$ and an epimorphism $H_{2}(\pi, \mathbb{Q}) \rightarrow H_{2}(G, \mathbb{Q})$ also induces a monomorphism modulo terms of the Harvey series. The Harvey series and associated Cochran-Harvey injectivity theorem underlie many recent applications of the von Neumann $\rho$-invariants to understanding concordance of knots and links, and homology cobordism of manifolds.

1.2. A new functorial commutator series and injectivity theorem. We consider the category $\mathcal{G}_{\Gamma}$ of groups $\pi$ over a fixed group $\Gamma$, i.e., $\pi$ is endowed with a homomorphism $\pi \rightarrow \Gamma$. (In the special case $\Gamma=\{e\}, \mathcal{G}_{\Gamma}$ canonically identifies with the category of groups.) In Section 3 , for a given coefficient ring $R$ we define a new series

$$
\pi \supset \pi^{(0)} \supset \pi^{(1)} \supset \cdots \supset \pi^{(n)} \supset \cdots
$$

of normal subgroups $\pi^{(n)}$ for each $\pi \in \mathcal{G}_{\Gamma}$ which we define in terms of the Bousfield $R \Gamma$-homology localization of groups and rings. We call this $\left\{\pi^{(n)}\right\}$ the Bousfield $R \Gamma$ local derived series to emphasize this is a functor on the category $\mathcal{G}_{\Gamma}$. Similarly, we define the Vogel-Cohn R $\Gamma$-local derived series using the Vogel localization of groups and Cohn localization of rings. Indeed the series can be defined in a more general situation; for a precise description of these local derived series, see Definition 3.2.

We prove that the $R \Gamma$-local derived series has the following properties:

Theorem. Let $\left\{\pi^{(n)}\right\}$ be the Bousfield (resp. Vogel-Cohn) $R \Gamma$-local derived series for $\pi$ in $\mathcal{G}_{\Gamma}$.

(1) (Functoriality) For any morphism $\pi \rightarrow G$ in $\mathcal{G}_{\Gamma}$, there are induced homomorphisms $\pi^{(n)} \rightarrow G^{(n)}$ and $\pi / \pi^{(n)} \rightarrow G / G^{(n)}$ for any $n$.

(2) (Injectivity) If $\pi \rightarrow G$ is a group homomorphism which is 2-connected on $H_{*}(-; R \Gamma)$ (resp. 2-connected on $H_{*}(-; R \Gamma)$ with $\pi$ finitely generated, $G$ finitely presented), then the induced map $\pi / \pi^{(n)} \rightarrow G / G^{(n)}$ is injective for any $n$.

The above theorem combines portions of Lemma 3.3 and Theorem 3.8 from the body of the paper.

In Section 5. we give computational examples which illustrate that our local derived series $\pi^{(n)}$ differs from the Harvey series and other derived series discussed below. In particular, we illustrate that $\pi / \pi^{(n)}$ may have torsion and/or infinite order elements, by comparison to the torsion free groups $\pi / \pi_{H}^{(n)}$.

The above two advances, regarding local commutator series and $L^{2}$-invariants, come together in the following theorem.

Theorem $\mathbf{7 . 2}$, Let $R$ be either $\mathbb{Z}_{p}, \mathbb{Z}_{(p)}$, or $\mathbb{Q}$. For a closed manifold $M$ over an amenable group $\Gamma$, view $\pi=\pi_{1}(M)$ as a group over $\Gamma$ and denote the $R \Gamma$-coefficient Vogel-Cohn local derived series of $\pi$ over $\Gamma$ by $\left\{\pi^{(n)}\right\}$. For the canonical map $\phi_{n}: \pi \rightarrow \pi / \pi^{(n)}$, the $L^{2}$-betti numbers $b_{i}^{(2)}\left(M, \phi_{n}\right)$ and the $L^{2}$-signature $\rho^{(2)}\left(M, \phi_{n}\right)$ are $R \Gamma$-homology cobordism invariants of $M$ for any $n<\infty$. In particular, when $\Gamma$ is trivial, $b_{i}^{(2)}\left(M, \phi_{n}\right)$ and $\rho^{(2)}\left(M, \phi_{n}\right)$ are always $R$-homology cobordism invariants. 
This applies to concordance of knots within a fixed homotopy class of a three manifold.

Corollary 1.1. (Compare to the Ph.D. thesis for Prudence Heck 20.) Let M be a three manifold with amenable fundamental group, let $g \in \Gamma=\pi_{1}(M)$. Let $\pi=\pi_{1}(M-K)$ for some knot $K \subset M$ with $K$ in the homotopy class $g$, and let $\pi^{(n)}$ be the $n^{\text {th }} R \Gamma$-local derived series subgroup. If $\pi^{(n)} / \pi^{(n+1)} \neq 0$, then there are infinitely many concordance classes of knots in the homotopy class of $g$ which for which no two are concordant to each other, and which are detected by the $L^{2}$ signature associated to $\pi \rightarrow \pi / \pi^{(n+1)}$.

Theorems concerning concordance of knots in general three manifolds and using $L^{2}$-methods were first explored and proven in the Ph.D. thesis of Prudence Heck [20]. The above argument follows hers, with Theorem 7.2 applied at the appropriate place in the argument, which the reader will find apparent. We refer the reader to this thesis 20 .

1.3. Comparison to other commutator series of groups. Let $R$ be a subring of the rationals or a finite cyclic ring. Recall that for a group $\pi$, the (non-local) $R$ coefficient derived series, or just $R$-derived series, is defined recursively as follows: $\pi^{0}=\pi$, and given $\pi^{n}$, then

$$
\pi^{n+1}=\operatorname{Ker}\left\{\pi^{n} \longrightarrow \frac{\pi^{n}}{\left[\pi^{n}, \pi^{n}\right]} \underset{\mathbb{Z}}{\otimes} R\right\}
$$

Here, $[\pi, \pi]$ is the usual commutator subgroup of the group $\pi$, the subgroup generated by commutators $[a, b]=a b a^{-1} b^{-1}$ where $a, b \in \pi$. When $R=\mathbb{Z}, \pi^{n}$ is the ordinary derived series. More generally, it is the fastest descending series such that successive quotients are abelian and $R$-torsion free. (This definition of this series directly parallels the $R$-lower central series of earlier authors, but was first applied in 19.$)$

To discuss alternative derived series of groups relevant in this work, we first discuss group localization.

In 2, Bousfield constructed the $H_{*}(-; R)$ localization of a space. Roughly, Bousfield associates to a space X, a space $E(X)$ (up to homotopy type) and a map of spaces $X \rightarrow E(X)$ which is initial with the property that given an homology equivalence $X \rightarrow Y$, there is a unique (homotopy class of a) map $Y \rightarrow E(X)$ making the following diagram homotopy commute. (A more general definition of localization is given in Section 2)

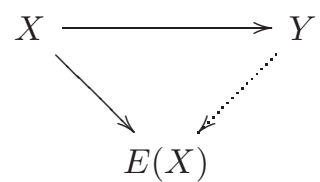

Bousfield showed that the group $\pi_{1}(E(X))$ depends only on the group $\pi_{1}(X)$. Thus, one can define a functor on the category of groups and group homomorphisms by $E(\pi)=\pi_{1}(E(B \pi))$, where $B \pi=K(\pi, 1)$ is the classifying space of the discrete group $\pi$. Bousfield showed that $\pi \rightarrow E(\pi)$ localizes groups and group homomorphisms with respect to $R$-homologically 2-connected homomorphisms; if $\pi \rightarrow G$ is $R$-homologically 2-connected, the induced map $E(\pi) \rightarrow E(G)$ is an isomorphism. Here $\pi \rightarrow G$ is said to be $R$-homologically 2-connected if the induced homomorphism $H_{i}(\pi ; R) \rightarrow H_{i}(G ; R)$ is an isomorphism for $i=1$ and an epimorphism for $i=2$.

Alternatively, in 29 Vogel defined a localization $\pi \rightarrow E(\pi)$ with respect to $R$ homologically 2-connected homomorphisms $\pi \rightarrow G$ with $\pi$ finitely generated and $G$ 
finitely presented. (See Section 2 for more details.) Vogel developed this variation of Bousfield's work to study homology types of compact manifolds, embeddings of compact manifolds, homology cobordism, and the Cappell-Shaneson homology surgery groups. (See also [3].)

The following series appears first in print in a paper of Cochran and Harvey 11 where they only consider Vogel group localization. In the definition below, $E(\pi)$ represents either Vogel or Bousfield localization of groups, yielding distinct functors.

Definition 1.2. The universal $R$-local derived series of a group $\pi$, denoted using braces, as $\pi^{\{n\}}$, is recursively defined as follows: $\pi^{\{0\}}=\pi$. Given the definition of $\pi^{\{n\}}$, define

$$
\pi^{\{n+1\}}=\operatorname{Ker}\left\{\pi^{\{n\}} \longrightarrow E(\pi)^{\{n\}} \longrightarrow \frac{E(\pi)^{\{n\}}}{\left[E(\pi)^{\{n\}}, E(\pi)^{\{n\}}\right]} \underset{\mathbb{Z}}{\otimes} R\right\} .
$$

We caution the reader that the universal $R$-local derived series does not equal, in general, the $R$-derived series defined in a prior paragraph. Since $E^{2}=E$, one can see easily that $E(\pi)^{\{n\}}=E(\pi)^{n}$ and $\pi^{\{n\}}$ is the preimage of $E(\pi)^{n}$ under $\pi \rightarrow E(\pi)$. In particular, the $R$-local and $R$-derived series agree for local groups.

One easily proves the following analogue of Stallings' theorem. Cochran and Harvey 11] first investigated this in depth, together with the above series, but the theorem below appears to have been known already to experts in the field. In particular, Cochran and Harvey state the Vogel case in terms of " $R$-closures" given in 6]. (See also 22.) We label it a folklore theorem, but wish to pay homage the aforementioned important contribution of Cochran and Harvey.

\section{Folklore Theorem.}

(1) (Bousfield case) Denote by $\pi^{\{n\}}$ the Bousfield universal R-local derived series. Suppose $\alpha: \pi \rightarrow G$ is R-homologically 2-connected. Then $\alpha$ induces an injection $\pi / \pi^{\{n\}} \rightarrow G / G^{\{n\}}$ for all $n$.

(2) (Vogel case) Denote by $\pi^{\{n\}}$ the Vogel universal $R$-local derived series. Suppose $\alpha: \pi \rightarrow G$ is $R$-homologically 2-connected with $\pi$ finitely generated and $G$ finitely presented. Then $\alpha$ induces an injection $\pi / \pi^{\{n\}} \rightarrow G / G^{\{n\}}$ for all $n$.

Proof. By construction $\pi / \pi^{\{n\}} \rightarrow E(\pi) / E(\pi)^{\{n\}}$ is injective for any $\pi$ and $n$. Since $E(\pi)=E(G)$, we have inclusions

$$
\pi / \pi^{\{n\}} \subset G / G^{\{n\}} \subset E(\pi) / E(\pi)^{\{n\}}=E(G) / E(G)^{\{n\}} .
$$

The Harvey series 10 may be thought of as a rational coefficient approximation to the Vogel universal local derived series, and the Cochran-Harvey injectivity theorem may be viewed as a Harvey series version of this Folklore Theorem. One issue with the universal local derived series is that the series as described is neither defined implicitly (that is it uses the construction of $E(\pi)$ in its definition) nor is it readily computable. By contrast, the Harvey series consists of subgroups of $\pi$ defined entirely from the structure of the group $\pi$ without making use of the group localization $E(\pi)$, and one can compute this in many useful circumstances.

On the other hand, a weakness of the Cochran-Harvey approach is that their series and the resulting quotients are not functorial under homomorphisms of groups. That is, a group homomorphism $\pi \rightarrow G$ does not induce $\pi_{H}^{(n)} \rightarrow G_{H}^{(n)}$ nor $\pi / \pi_{H}^{(n)} \rightarrow G / G_{H}^{(n)}$ in general.

The $R \Gamma$-local series of this paper might be viewed as a compromise theory one that lies closer to the ideal (i.e., the universal local derived) series, that works for a wider range of (twisted) coefficient systems, that is computable, that provides an injectivity theorem, and importantly, that is functorial. 
For $\Gamma=\{e\}$, the Vogel-Cohn local derived series refines the Harvey series and lies closer to the universal local derived series, as the following comparison theorem shows.

Theorem 4.1. Let $\Gamma=\{e\}$ and $\left\{\pi^{(n)}\right\}$ be the Vogel-Cohn $R \Gamma$-local derived series of a group $\pi$. Let $\left\{\pi^{n}\right\},\left\{\pi^{\{n\}}\right\},\left\{\pi_{H}^{(n)}\right\}$ be the $R$-derived series, Vogel universal $R$-local derived series, and the Harvey series, respectively. Then, for any $\pi$ and $n$, we have

$$
\pi^{n} \subset \pi^{\{n\}} \subset \pi^{(n)} \subset \pi_{H}^{(n)}
$$

In a future paper we will combine our results on homology with $L^{2}$-coefficients (Theorem 6.6 and its applications), the universal $R \Gamma$-local derived series, and some computations using compact, orientable, three manifold groups whose group localization contains torsion, to obtain further examples of homology equivalent three manifolds which are not homology cobordant.

1.4. Organization of the paper. In the first half of this paper (Sections 2 5), we define and investigate the $R \Gamma$-local derived series. In Section 2 , we give necessary preliminaries on localizations of groups and rings. In Section 3 , we define the $R \Gamma$-local derived series and prove its functoriality and the injectivity theorem. In Section 4 we compare the local derived series with related commutator-type series, including the Harvey series. In Section 5 we give some computational examples of the local derived series which will be used in later applications.

In the latter half of this paper (Sections 6 8) we study homological properties of $L^{2}$-theory and applications to manifolds. In Section 6 we prove our main theorem, the " $L^{2}$-dimensional" local property of the von Neumann group ring of an amenable group, which play a crucial role in our applications of the local derived series to manifolds. For those most interested in this result, one may read this section independently of the prior sections. In Section 7 we investigate homology cobordism invariance of $L^{2}$-signature defects associated to quotients of local derived series of fundamental groups. In Section 8 we give some applications, including Theorems 8.2 Corollary 8.5, and some generalizations.

Acknowledgements. This work was partly supported by a Korea Science and Engineering Foundation grant funded by the Korean government (MOST) (R012007-000-11687-0) and by a Korea Research Foundation grant funded by the Korean Government (MOEHRD) (KRF-2007-412-J02302). The second author was supported by NSF grant DMS-0707078. The first author thanks the Department of Mathematics, Indiana University, for supporting his visit in 2008 summer.

\section{LOCALIZATIONS OF GROUPS AND RINGS}

In this section we discuss basic definitions and properties of homology localizations of groups and rings, which are essentially due to Bousfield, Vogel, and Cohn. Theorems and propositions in this section are not new and have been widely known (at least to experts in related fields). We will focus on those facts that we will need in later sections.

We begin with the general definition of a localization functor in a category.

Definition 2.1. Suppose $\Omega$ is a collection of morphisms in a category $\mathcal{C}$.

(1) An object $A$ in $\mathcal{C}$ is local with respect to $\Omega$ if for any $\pi \rightarrow G$ in $\Omega$ and for any $\pi \rightarrow A$, there exists a unique morphism $G \rightarrow A$ making the following 
diagram commute:

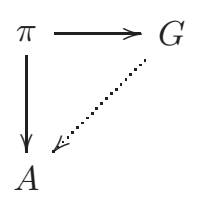

(2) A localization functor with respect to $\Omega$ is a functor

$$
E: \mathcal{C} \longrightarrow\{\text { local objects in } \mathcal{C}\}
$$

endowed with a natural transformation $\left\{p_{G}: G \rightarrow E(G)\right\}_{G \in \mathcal{C}}$ such that for any morphism $G \rightarrow A$ into a local object $A$, there is a unique morphism $E(G) \rightarrow A$ making the following diagram commute:

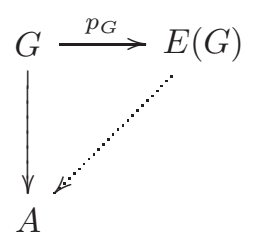

The following statements are consequences of the universal properties stated above. We omit proofs.

\section{Proposition 2.2.}

(1) A localization functor is unique (up to a natural equivalence).

(2) A localization functor $E$ is an idempotent, i.e., $E^{2}=E$.

(3) If $E$ is a localization functor with respect to $\Omega$, then every $\pi \rightarrow G$ in $\Omega$ induces an equivalence $E(\pi) \rightarrow E(G)$.

2.1. Localizations of groups. We will give a very brief introduction to a homology localization theory of groups due to Bousfield and Vogel. Let $\mathcal{G}_{\Gamma}$ be the category of groups over a fixed group $\Gamma$. Precisely, the objects of $\mathcal{G}_{\Gamma}$ are homomorphisms of a group $\pi$ into $\Gamma$. A morphism from $\pi \rightarrow \Gamma$ to $G \rightarrow \Gamma$ is a homomorphism $\pi \rightarrow G$ making the following diagram commute:

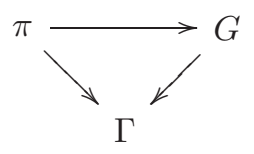

We abuse notation and denote an object $\pi \rightarrow \Gamma$ of $\mathcal{G}_{\Gamma}$ by $\pi$.

As a special case, when $\Gamma$ is a trivial group, $\mathcal{G}_{\Gamma}$ is canonically equivalent to the category of groups.

Let $R$ be either a finite cyclic ring $\mathbb{Z}_{d}$ or a subring of $\mathbb{Q}$. For an object $\pi$ in $\mathcal{G}_{\Gamma}$, the homology $H_{*}(\pi ; R \Gamma)$ with local coefficients is defined, where $R \Gamma$ denotes the group ring of $\Gamma$ over $R$. We will consider localizations with respect to $R \Gamma$ coefficient homology. Specifically, let $H R$ be the collection of morphisms $\alpha: \pi \rightarrow G$ in $\mathcal{G}_{\Gamma}$ which are 2 -connected on $H_{*}(-; R \Gamma)$, that is, $\alpha$ induces an isomorphism on $H_{1}(-; R \Gamma)$ and an epimorphism on $H_{2}(-; R \Gamma)$. A localization functor on $\mathcal{G}_{\Gamma}$ with respect to $H R$ is called the Bousfield HR-localization.

Regarding applications to geometric topology (especially to the study of compact manifolds), one is naturally led to consider the subcollection of morphisms on finitely presented groups. Motivated by this, we also consider the subcollection $\Omega_{0}$ of morphisms $\alpha: \pi \rightarrow G$ in $H R$ with $\pi$ and $G$ finitely presented. A localization functor on $\mathcal{G}_{\Gamma}$ with respect to $\Omega_{0}$ is called the Vogel localization. 
The following results are essentially due to Bousfield [1, 2] and Vogel [29. (See also Farjoun-Orr-Shelah [17, Levine [22, and Cha [6] for equation-based approaches. Levine appears to have first observed these combinatorial approaches within the context of Vogel localization, with Farjoun and Shelah doing so later but independently in the context of Bousfield's work.)

Theorem 2.3 (Bousfield [2, 1], Vogel 29]). For any $R$ and $\Gamma$, there exist a Bousfield $H R$-localization functor and a Vogel localization functor on $\mathcal{G}_{\Gamma}$.

In the case of the Vogel localization $E$, the map $\pi \rightarrow E(\pi)$ is not always contained in $\Omega_{0}$, even when $\pi$ is finitely presented. In this case, one can enlarge $\Omega_{0}$ to a collection, $\bar{\Omega}_{0}$ which contains $\pi \rightarrow E(\pi)$, and for which the resulting localization functors agree on classes in $\Omega_{0}$. Let $\bar{\Omega}_{0}$ be the collection of morphisms $\pi \rightarrow G$ such that for any given commutative diagram

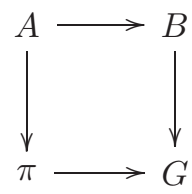

of morphisms in $\mathcal{G}_{\Gamma}$ with $A$ and $B$ are finitely presented, there is $A_{0} \rightarrow B_{0}$ in $\Omega_{0}$ which fits into the following commutative diagram:

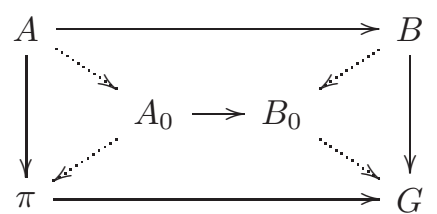

Remark 2.4. It is easily seen that $\bar{\Omega}_{0} \subset H R$, since any homology class of a free chain complex is supported by a finitely generated subcomplex.

Theorem 2.5 (Bousfield [2, 1, Vogel [29]).

(1) For the Bousfield HR-localization $E, \pi \rightarrow E(\pi)$ is in $H R$ for all $\pi \in \mathcal{G}_{\Gamma}$.

(2) The Vogel localization $E$ is a localization with respect to $\bar{\Omega}_{0}$, and in this case, $\pi \rightarrow E(\pi)$ is in $\bar{\Omega}_{0}$ for all $\pi \in \mathcal{G}_{\Gamma}$.

We sketch the proofs of Theorems 2.3 and 2.5 in an appendix for the convenience of our readers.

Remark 2.6. In known applications of the localization theory to the study of manifold embeddings, one is naturally led to consider localizations with respect to certain morphisms $\alpha: \pi \rightarrow G$ in $\Omega$ with the property that $\operatorname{Ker}\{G \rightarrow \Gamma\}$ is normally generated by the image of $\operatorname{Ker}\{\pi \rightarrow \Gamma\}$. (See, for instance, [21.) It is known that Theorems 2.3 and 2.5 also hold for the analogues of $H R, \Omega_{c}$ and $\bar{\Omega}_{c}$ with this normal generation condition. It can also be seen that all results in this paper hold under the normal generation condition.

Remark 2.7. Following Vogel, one may also consider only homomorphisms $\pi \rightarrow G$ in $H R$ such that the $\pi$ and $G$ have a number of generators and relations each bounded below by a fixed cardinal, $c$. For instance, the Vogel localization of groups is localization with respect to $H R$ homomorphisms and the cardinal $c=\aleph_{0}$. However, one can show that for any cardinal $c>\aleph_{0}$, the resulting localization equals the usual Bousfield $H R$ localization. Analogous observations apply equally to the (module and) ring localizations which will de discussed below. 
2.2. Localizations of rings. We begin by recalling Cohn's classical ring localization. Let $R$ and $S$ be rings with unity, together with a ring homomorphism $\epsilon: R \rightarrow S$.

The Cohn localization, $\Lambda$, of the homomorphism $\epsilon: R \rightarrow S$ is a ring endowed with a ring homomorphism $R \rightarrow \Lambda$ satisfying the following conditions:

(1) Given any square matrix $A$ over $R$, then $A \otimes_{R} S$ in invertible if and only of $A \otimes_{R} \Lambda$ is invertible, and

(2) $R \rightarrow \Lambda$ is initial among homomorphisms of $R$ satisfying (1), that is, if $R \rightarrow \Lambda^{\prime}$ is another ring homomorphism satisfying (1), then there is a unique ring homomorphism $\Lambda \rightarrow \Lambda^{\prime}$ making the following diagram commute:

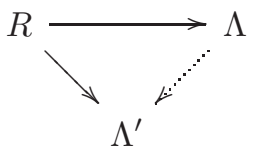

In [16], Cohn constructed this localization and showed uniqueness and functoriality up to isomorphism. We give an alternative description of Cohn localization here as a localization of modules with a naturally imposed ring structure. (Of particular interest in this paper is the case of $R \pi \rightarrow R \Gamma$ induced by a homomorphism $\pi \rightarrow \Gamma$ in $\mathcal{G}_{\Gamma}$.)

Let $\mathcal{W}$ be the collection of homomorphisms $\alpha: F \rightarrow F^{\prime}$ between (right) free $R$-modules $F$ and $F^{\prime}$ with the same rank such that

$$
\alpha \otimes 1_{S}: F \otimes_{R} S \longrightarrow F^{\prime} \otimes_{R} S
$$

is an isomorphism. (Note that the rank of $F$ and $F^{\prime}$ may be an arbitrary cardinal.) Recall that, with respect to $\mathcal{W}$, a local $R$-module is defined to be an $R$-module such that for any $\alpha: F \rightarrow F^{\prime}$ in $\mathcal{W}$,

$$
\alpha^{*}: \operatorname{Hom}\left(F^{\prime}, M\right) \longrightarrow \operatorname{Hom}(F, M)
$$

is an isomorphism. A localization functor with respect to $\mathcal{W}$

$$
E:\{R \text {-modules }\} \longrightarrow\{\text { local } R \text {-modules }\}
$$

in the sense of Definition 2.1 is called the Bousfield $H \mathbb{Z}$-localization functor. (See Remark 2.9])

We also consider module localization with an additional finite rank condition: let $\mathcal{W}_{0}$ be the collection of $\alpha: F \rightarrow F^{\prime}$ in $\mathcal{W}$ such that $\operatorname{rank} F=\operatorname{rank} F^{\prime}$ is finite. We call the resulting localization functor of $R$-modules the Cohn localization. The following existence results are essentially due to Bousfield and Cohn:

Theorem 2.8 (Bousfield [2], Cohn [16]). For any $R \rightarrow S$, there exist the Bousfield $H \mathbb{Z}$-localization and Cohn localization of modules.

The Bousfield $H \mathbb{Z}$ and Cohn localization of modules have the following properties:

(1) If $E$ represents either the Bousfield $H \mathbb{Z}$ and Cohn localization functor of $R$-modules, then the $R$-module $E(R)$ admits a natural ring structure such that $R \rightarrow E(R)$ is a ring homomorphism.

(2) The Bousfield $H \mathbb{Z}$-localization $E(R)$ has the following universal property as a ring: let $\mathcal{C}^{\mathcal{W}}$ be the category of rings $\Lambda$ endowed with a ring homomorphism $R \rightarrow \Lambda$ such that for any $\alpha: F \rightarrow F^{\prime}$ in $\mathcal{W}$,

$$
\alpha \otimes 1_{\Lambda}: F \otimes_{R} \Lambda \longrightarrow F^{\prime} \otimes_{R} \Lambda
$$

is an isomorphism. Then $E(R)$ is the initial object of $\mathcal{C}^{\mathcal{W}}$.

(3) The Cohn module localization of $R$ with the ring structure from (1) has the universal property analogous to $(2)$ where $\mathcal{W}_{0}$ plays the role of $\mathcal{W}$. 
We give proofs in an appendix. Property (3) says that the Cohn localization of the $R$-module $R$ agrees with the aforementioned Cohn localization of the ring $R$ (endowed with $\epsilon: R \rightarrow S$ ). In parallel to this, we call $E(R)$ in (2) the Bousfield $H \mathbb{Z}$-localization of the ring $R$ endowed with $\epsilon: R \rightarrow S$. We note that this viewpoint enables us to regard both ring localizations as special cases of Definition 2.1 and to use standard properties of a localization functor.

Remark 2.9. In [2], Bousfield originally defined $H \mathbb{Z}$-localization of $R \pi$-modules for $R \pi \rightarrow R \Gamma$ induced by a group homomorphism $\pi \rightarrow \Gamma$. He localizes with respect to the class of $R \pi$-module homomorphisms $\alpha: A \rightarrow B$ such that $\operatorname{Tor}_{i}^{R \pi}(R \Gamma, A) \rightarrow$ $\operatorname{Tor}_{i}^{R \pi}(R \Gamma, B)$ is an isomorphism for $i=0$ and a surjection for $i=1$. He dealt mainly with the case of $\Gamma=\{e\}$ and $R=\mathbb{Z}$, so that the terms $\operatorname{Tor}_{i}^{R \pi}(R \Gamma,-)$ equal $H_{i}(\pi ;-)$, but his arguments extend to the case of any $\Gamma$ and $R$. This module localization agrees with the module localization with respect to $\mathcal{W}$ described above. We prove this in an appendix.

We note that by applying the universal property of the Bousfield $H \mathbb{Z}$ (or Cohn) localization $\Lambda$ for $\epsilon: R \rightarrow S$, there is a unique homomorphism $\Lambda \rightarrow S$ such that the following diagram commutes:

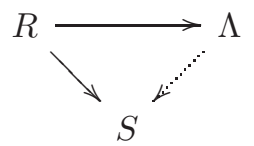

The Bousfield $H \mathbb{Z}$ (resp. Cohn) localization is the initial object of the subcategory of objects of $\mathcal{C}^{\mathcal{W}}$ (resp. $\left.\mathcal{C}^{\mathcal{W}_{0}}\right)$ admitting such a diagram.

Notation 2.10. Given $\pi \rightarrow \Gamma$ an element of the category $\mathcal{G}_{\Gamma}$, we denote the Cohn and Bousfield $H \mathbb{Z}$-localization of $R \pi$ (endowed with $R \pi \rightarrow R \Gamma$ ) by $L(\pi)=\Lambda$ and observe that this is functorial on morphisms in $\mathcal{G}_{\Gamma}$.

2.3. Ring localizations and homology. The following property of the ring localizations will play a key role later. It is essentially due to Vogel.

Theorem 2.11 (Vogel [30]). Suppose $\pi \rightarrow G$ is a morphism in $\mathcal{G}_{\Gamma}$.

(1) The Bousfield $H \mathbb{Z}$-localization $\Lambda$ of $R G$ satisfies the following: every $\pi \rightarrow G$ in $H R$ is 2-connected on $H_{*}(-; \Lambda)$.

(2) The Cohn localization $\Lambda$ of $R G$ satisfies the following: every $\pi \rightarrow G$ in $\bar{\Omega}_{0}$ is 2-connected on $H_{*}(-; \Lambda)$.

Proof. We first prove (2). Suppose $\pi \rightarrow G$ is in $\bar{\Omega}_{0}$. Let $C_{*}(G, \pi ; \Lambda)$ be the $\Lambda$ coefficient $\mathrm{CW}$ chain complex of the mapping cylinder $M$ of $K(\pi, 1) \rightarrow K(G, 1)$. All CW-complexes are over $K(\Gamma, 1)$.

For a cycle $z$ in $C_{i}(G, \pi ; \Lambda)(i=1,2)$, there is a finite subpair of $(M, K(\pi, 1))$ in which $z$ is supported. Therefore, we obtain the following commutative diagram in $\mathcal{G}_{\Gamma}$

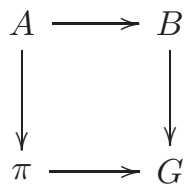


such that $A$ and $B$ are the finitley presented fundamental groups of this subpair and $z$ is the image of some cycle in $C_{*}(B, A ; \Lambda)$. Since $\pi \rightarrow G$ is in $\bar{\Omega}_{0}$, we obtain

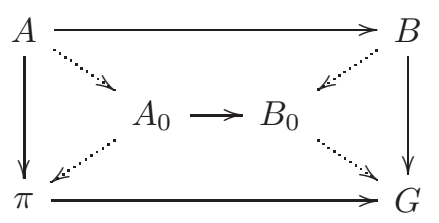

where $A_{0} \rightarrow B_{0}$ is a morphism in $\Omega_{0}$.

Since $z$ is the image of a cycle in $C_{*}\left(B_{0}, A_{0} ; \Lambda\right)$, in order to conclude $z$ bounds it suffices to prove the following claim: $H_{i}\left(B_{0}, A_{0} ; \Lambda\right)=0$ for $i=1,2$. Since $C_{i}\left(B_{0}, A_{0} ; R G\right)$ can be assumed to be $R G$-free module of finite rank for $i \leq 2$, the claim is proved by a standard partial chain contraction argument, which is originally due to Vogel. (For example, refer to [30, 23], [14.) This completes the proof of (2).

The proof of (1) is similar but easier since we do not have to consider $A \rightarrow B$ and $A_{0} \rightarrow B_{0}$; we can directly apply the above chain contraction argument to $C_{*}(G, \pi ; R \pi)$ to show that $H_{i}(G, \pi ; \Lambda)=0$ for $i=1,2$.

\section{LOCAL DERIVED SERIES AND INJECTIVITY}

Fix $R$ and let $\mathcal{G}_{\Gamma}$ be as before. In this section we consider a collection $\Omega$ of morphisms in $\mathcal{G}_{\Gamma}$, a localization functor $E$ on $\mathcal{G}_{\Gamma}$, and a localization functor $L$ for group rings; suppose $(\Omega, E, L)$ is either one of the following two cases:

$$
\text { (i) }\left\{\begin{array}{l}
\Omega=H R \\
E=\text { Bousfield } H R \text {-localization } \\
L=\text { Bousfield } H \mathbb{Z} \text {-localization }
\end{array}\right\} \text { or (ii) }\left\{\begin{array}{l}
\Omega=\bar{\Omega}_{0} \\
E=\text { Vogel localization } \\
L=\text { Cohn localization }
\end{array}\right\}
$$

We will define a commutator-type series for each $\pi \in \mathcal{G}_{\Gamma}$ which is determined by $R$ and $(\Omega, E, L)$ and prove that it admits an injectivity theorem. Indeed all the properties of $(\Omega, E, L)$ we need are the following, which are easily verified for the above cases (i) and (ii), by our discussion in the previous section (in particular see Theorems 2.5 and 2.11):

(1) $E$ is a localization functor on $\mathcal{G}_{\Gamma}$ with respect to $\Omega$.

(2) $\pi \rightarrow E(\pi)$ is in $\Omega$ for any $\pi \in \mathcal{G}_{\Gamma}$.

(3) Every $\pi \rightarrow G$ in $\Omega$ is 2-connected on $H_{*}(-; \Lambda)$, where $\Lambda=L(G)$.

Definition 3.1. We call $(\Omega, E, L)$ a homology localization triple if the above (1), (2), and (3) hold. We call the homology localization triples (i) and (ii) described above the Bousfield localization triple and Vogel-Cohn localization triple, respectively.

Throughout this section, $(\Omega, E, L)$ is always assumed to be a homology localization triple, and $\widehat{\pi}$ denotes $E(\pi)$ for $\pi \in \mathcal{G}_{\Gamma}$.

\section{1. $R \Gamma$-local derived series.}

Definition 3.2. For each $\pi$ in $\mathcal{G}_{\Gamma},(\Omega, E, L)$ a homology localization triple, and for each ordinal $n$, the $R \Gamma$-local derived series $\left\{\pi^{(n)}\right\}$ is defined transfinite-inductively as follows:

$$
\pi^{(0)}=\operatorname{Ker}\{\pi \longrightarrow \Gamma\},
$$

and assuming $\pi^{(n)}$ has been defined, $\pi^{(n+1)}$ is defined by

$$
\pi^{(n+1)}=\operatorname{Ker}\left\{\pi^{(n)} \longrightarrow \frac{\pi^{(n)}}{\left[\pi^{(n)}, \pi^{(n)}\right]}=H_{1}\left(\pi ; \mathbb{Z}\left[\frac{\pi}{\pi^{(n)}}\right]\right) \longrightarrow H_{1}\left(\pi ; \Lambda_{\pi}\right)\right\}
$$


where $\Lambda_{\pi}$ designates the ring localization $L\left(\widehat{\pi} / \widehat{\pi}^{(n)}\right)$, and recall that $\widehat{\pi}=E(\pi)$. For a limit ordinal $n$, define $\pi^{(n)}=\bigcap_{k<n} \pi^{(k)}$.

By an induction one easily verifies that each $\pi^{(n)}$ is a normal subgroup of $\pi$ forming a normal series

$$
\pi \supset \pi^{(0)} \supset \pi^{(1)} \supset \cdots \supset \pi^{(n)} \supset \cdots .
$$

Lemma 3.3. For any $n$, the associations $\pi \rightarrow \pi^{(n)}, \pi \rightarrow \pi / \pi^{(n)}$ are functors $\mathcal{G}_{\Gamma} \rightarrow \mathcal{G}_{\Gamma}$. In particular, any morphism $\pi \rightarrow G$ in $\mathcal{G}_{\Gamma}$ induces group homomorphisms $\pi^{(n)} \rightarrow G^{(n)}$ and $\pi / \pi^{(n)} \rightarrow G / G^{(n)}$.

Proof. We use a transfinite induction on $n$ to show that a morphism $\pi \rightarrow G$ gives rise to a group homomorphism on the normal subgroups $(-)^{(n)}$ and so on their quotients. For $n=0$, the conclusion is obvious. Assume that the conclusion holds for $n$. Let $\Lambda_{\pi}=L\left(\widehat{\pi} / \widehat{\pi}^{(n)}\right)$ and $\Lambda_{G}=L\left(\widehat{G} / \widehat{G}^{(n)}\right)$ as before. Then since $L$ is functorial, there is an induced map $\Lambda_{\pi} \rightarrow \Lambda_{G}$. So we have a commutative diagram

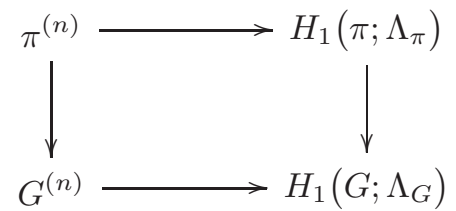

where the horizontal maps are the compositions appearing in the definition of $\pi^{(n+1)}$ and $G^{(n+1)}$. From this it follows that $\pi^{(n+1)}$ is sent into $G^{(n+1)}$.

For a limit ordinal $n$, assuming the conclusion holds for $k<n$, the conclusion for $n$ follows immediately by taking the limit.

Remark 3.4. The above naturality is essentially due to the functoriality of $L$ and $E$ used in the above definition. By contrast, the Harvey series $\left\{\pi_{H}^{(n)}\right\}$ defined in [10] is not functorial on the category of groups since the Ore localization of group rings is not functorial.

The following Lemma is proven easily from the definition of the local derived series.

Lemma 3.5. Suppose $(\Omega, E, L)$ and $\left(\Omega^{\prime}, E^{\prime}, L^{\prime}\right)$ are homology localization triples (possibly for distinct $R$ ) and there are natural transformations $E \rightarrow E^{\prime}$ and $L \rightarrow L^{\prime}$. Then the local derived series determined by $(\Omega, E, L)$ is contained in the local derived series determined by $\left(\Omega^{\prime}, E^{\prime}, L^{\prime}\right)$.

Definition 3.6. We have the following special cases of Definition 3.2 using the Bousfield and Vogel-Cohn homology localization triples, cases (i) (resp. (ii)) described in the beginning of section 3 . We denote this series $\left\{\pi^{(n)}\right\}$ and call it the Bousfield (resp. Vogel-Cohn) RГ-local derived series of $\pi$ over $\Gamma$.

\section{Proposition 3.7.}

(1) If $R \subset R^{\prime}$, then the $R$-coefficient Bousfield local derived series is contained in the $R^{\prime}$-coefficient Bousfield local derived series. Similarly for the VogelCohn case.

(2) For any $R \subset \mathbb{Q}$, the Vogel-Cohn local derived series is contained in the Bousfield local derived series.

The existence of the required natural transformations follows from the universal property of a localization and the facts $\Omega_{0} \subset H R$ and $\mathcal{W}_{0} \subset \mathcal{W}$. We omit details. 


\subsection{An injectivity theorem.}

Theorem 3.8. Suppose $(\Omega, E, L)$ is a homology localization triple and let $\left\{\pi^{(n)}\right\}$ be the associated local derived series. Then for any $\pi \rightarrow G$ in $\Omega$ and for any ordinal $n$, the induced homomorphism $\pi / \pi^{(n)} \rightarrow G / G^{(n)}$ is injective. In particular, $\pi / \pi^{(n)} \rightarrow \widehat{\pi} / \widehat{\pi}^{(n)}$ is injective.

Proof. We use a transfinite induction on $n$ to prove the first statement. Once we prove this, the second statement follows immediately since $\pi \rightarrow \widehat{\pi}$ is in $\Omega$.

Since

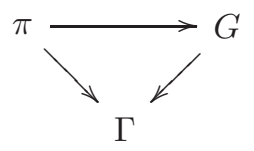

commutes, $\pi^{(0)}=\operatorname{Ker}\{\pi \rightarrow \Gamma\}$ is exactly the inverse image of $G^{(0)}=\operatorname{Ker}\{G \rightarrow \Gamma\}$. Therefore the conclusion holds for $n=0$.

Suppose it holds for $n$. Denote $L\left(\widehat{\pi} / \widehat{\pi}^{(n)}\right)$ by $\Lambda_{\pi}$ as before. To show the conclusion for $n+1$, consider the commutative diagram

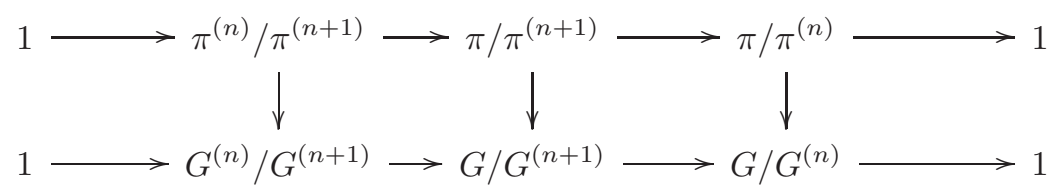

with exact rows. By the five lemma and the induction hypothesis, it suffices to show the injectivity of the homomorphism $\pi^{(n)} / \pi^{(n+1)} \rightarrow \mathcal{G}^{(n)} / G^{(n+1)}$.

Since $\pi \rightarrow G$ is in $\Omega$, we have $\widehat{\pi} \cong \widehat{G}$ and $\Lambda_{\pi} \cong \Lambda_{G}$. Therefore, we have the following commutative diagram, where $\pi^{(n+1)}$ and $G^{(n+1)}$ are the kernels of the horizontal arrows:

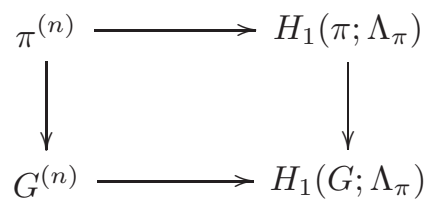

By the defining properties of a homology localization triple, $\pi \rightarrow G$ is 2-connected on $H_{*}(-; \Lambda)$ where $\Lambda=L(\pi)$. Since there is a natural homomorphism $\Lambda \rightarrow \Lambda_{\pi}$ by the naturality of the ring localization $L, \pi \rightarrow G$ is also 2-connected on $H_{*}\left(-; \Lambda_{\pi}\right)$. Consequently the rightmost vertical arrow in the above diagram is an isomorphism. From this it follows that the inverse image of $G^{(n+1)} \subset G^{(n)}$ under $\pi^{(n)} \rightarrow G^{(n)}$ is exactly $\pi^{(n+1)}$. This proves the conclusion for $n+1$. The limit ordinal case follows by taking limits.

\section{Remark 3.9.}

(1) Consider the case of the rational Bousfield or Vogel-Cohn derived series over $\Gamma=\{e\}$. Then as a part of the hypotheses of Theorem 3.8, we have an $H_{1}$-condition that $\pi \rightarrow G$ induces an isomorphism on $H_{1}(-; \mathbb{Q})$. This can be weakened to only require that the induced map on $H_{1}(-; \mathbb{Q})$ be injective. For, one can think of an appropriate map from the free product $\pi * F$ with a free group $F$ into $G$ which is 2-connected, so that $(\pi * F) /(\pi *$ $F)^{(n)} \rightarrow G / G^{(n)}$ is injective by Theorem 3.8. Since $\pi \rightarrow \pi * F$ has a left inverse, so does $\pi / \pi^{(n)} \rightarrow(\pi * F) /(\pi * F)^{(n)}$ by functoriality. It follows that $\pi / \pi^{(n)} \rightarrow G / G^{(n)}$ is injective.

(2) For the Vogel-Cohn derived series over $\Gamma=\{e\}$ (for any $R$ ), one can prove the following Dwyer-type injectivity theorem, which is similar to a result 
in 12]: if a homomorphism $\pi \rightarrow G$ between finitely presented groups induces an isomorphism $\widehat{\pi} \cong \widehat{G}$ and an epimorphism

$$
H_{2}(\pi ; R) \longrightarrow H_{2}(G) / \operatorname{Im}\left\{H_{2}\left(G^{(n)} ; R\right) \longrightarrow H_{2}(G ; R)\right\},
$$

then $\pi / \pi^{(n+1)} \rightarrow G / G^{(n+1)}$ is injective. The proof is omitted.

\section{Comparison With Harvey series}

In this section, for $\Gamma=\{e\}$, we compare the $R \Gamma$-local derived series with the Harvey series 10 and other related series. Because we need to distinguish several series, we use the following notation (temporarily): Fix a coefficient ring $R$, and for a group $\pi$, let $\left\{\pi^{n}\right\},\left\{\pi^{\{n\}}\right\}$, and $\left\{\pi^{(n)}\right\}$ be the $R$-coefficient derived series (defined immediately above Definition 3), universal local derived series (see Defintion 3), and Vogel-Cohn $R$-local derived series (see Definition 3.6). (In defining $\pi^{\{n\}}$ and $\pi^{(n)}$ we assume $\Gamma=\{e\}$ so that the series are defined for any group $\pi$.) Let $\left\{\pi_{H}^{(n)}\right\}$ be the Harvey derived series [10].

Theorem 4.1. For any group $\pi$ and for any $n, \pi^{n} \subset \pi^{\{n\}} \subset \pi^{(n)} \subset \pi_{H}^{(n)}$.

Proof. We use an induction on $n$. For $n=0$, the conclusion holds since the initial (zeroth) terms of all the concerned series are $\pi$ itself by definition. Suppose the conclusion holds for $n$. Let $L(G)$ be the Cohn localization of the augmentation map $R G \rightarrow R$. For a group $G$ such that $\mathbb{Z} G$ is an Ore domain, we denote by $\mathcal{K}(G)$ the Ore localization $\mathbb{Z} G(\mathbb{Z} G-\{0\})^{-1}$ of $\mathbb{Z} G$. (In this proof $\mathcal{K}(G)$ is always well-defined whenever the notation $\mathcal{K}(G)$ is used.)

We consider the following commutative diagram; note that, by definition, the $(n+1)$-st terms of the concerned series equal the kernels of the rows. (To see that the kernel of the second row is $\pi^{\{n+1\}}=\operatorname{Ker}\left\{\pi \rightarrow \widehat{\pi} / \widehat{\pi}^{(n+1)}\right\}$, note that $\widehat{\pi}^{\{n\}}=\widehat{\pi}^{n}$.)

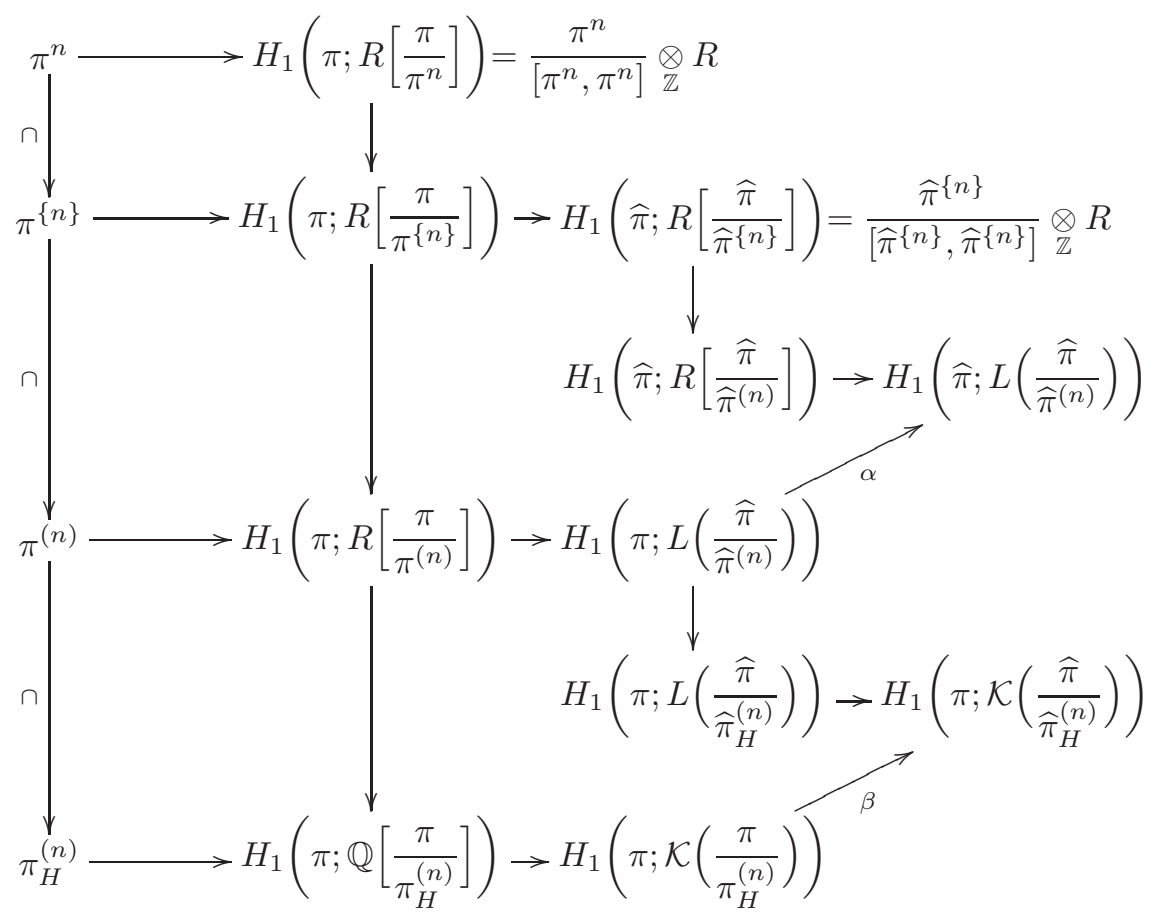

Note that in order to obtain the diagram we need the existence of the following natural maps: 
(1) $L\left(\widehat{\pi} / \widehat{\pi}_{H}^{(n)}\right) \rightarrow \mathcal{K}\left(\widehat{\pi} / \widehat{\pi}_{H}^{(n)}\right)$. Since $\widehat{\pi} / \widehat{\pi}_{H}^{(n)}$ is known to be a poly-torsion-freeabelian group [10], from Strebel's result 28] it follows that the morphism $R\left[\widehat{\pi} / \widehat{\pi}_{H}^{(n)}\right] \rightarrow \mathcal{K}\left(\widehat{\pi} / \widehat{\pi}_{H}^{(n)}\right)$ is an object of the category $\mathcal{C}^{\mathcal{W}}$ we described in Subsection 2.2 in order to define the Cohn localization. Therefore, since $L\left(\widehat{\pi} / \widehat{\pi}_{H}^{(n)}\right)$ is an initial object of $\mathcal{C}^{\mathcal{W}_{0}}$, the desired map exists.

(2) $\mathcal{K}\left(\pi / \pi_{H}^{(n)}\right) \rightarrow \mathcal{K}\left(\widehat{\pi} / \widehat{\pi}_{H}^{(n)}\right)$. Since $\pi / \pi_{H}^{(n)} \rightarrow \widehat{\pi} / \widehat{\pi}_{H}^{(n)}$ is injective [10, 6], it induces an injection $\mathcal{K}\left(\pi / \pi_{H}^{(n)}\right) \rightarrow \mathcal{K}\left(\widehat{\pi} / \widehat{\pi}_{H}^{(n)}\right)$ between (skew) fields.

Observe the following:

(1) $\alpha$ is an isomorphism by Theorems 2.5 and 2.11

(2) $\beta$ is injective since any $\mathcal{K}\left(\pi / \pi_{H}^{(n)}\right)$-module, especially $H_{1}\left(\pi ; \mathcal{K}\left(\pi / \pi_{H}^{(n)}\right)\right)$, is free and $\mathcal{K}\left(\widehat{\pi} / \widehat{\pi}_{H}^{(n)}\right)$ is flat over its sub(skew)field $\mathcal{K}\left(\pi / \pi_{H}^{(n)}\right)$.

Using the above observations, the desired result for $n+1$ follows from a straightforward diagram chase. The limit ordinal case is immediate.

The next result deals with the special case of $R=\mathbb{Z}_{p}$.

Theorem 4.2. Suppose $R=\mathbb{Z}_{p}$ and $\pi$ is finitely generated. Then $\pi^{n}=\pi^{\{n\}}=\pi^{(n)}$ and $\pi / \pi^{n} \cong \widehat{\pi} / \widehat{\pi}^{n}$ for $n<\infty$.

To prove this we need:

Lemma 4.3 (Strebel, Levine). For any finite p-group $G$, the Cohn localization of the group ring $\mathbb{Z}_{p} G$ is $\mathbb{Z}_{p} G$ itself.

Lemma 4.3 follows immediately from Strebel's work 28 or Levine's argument used in the proof of [23, Lemma 4.3, p89]. We omit details.

Proof of Theorem 4.2. We will show that $\pi^{n}=\pi^{(n)}$ and $\pi / \pi^{n} \cong \widehat{\pi} / \widehat{\pi}^{n}$ by an induction on $n$. For $n=0$, everything holds obviously. Suppose the desired conclusion holds for $n$. We have

$$
\pi^{(n+1)}=\operatorname{Ker}\left\{\pi^{n} \longrightarrow H_{1}\left(\pi ; \mathbb{Z}_{p}\left[\frac{\pi}{\pi^{n}}\right]\right) \longrightarrow H_{1}\left(\pi ; L\left(\frac{\pi}{\pi^{n}}\right)\right)\right\} .
$$

It is known that $\pi / \pi^{n}$ is a finite $p$-group if $\pi$ is finitely generated (e.g., see [9].) By Lemma 4.3 stated above, $L\left(\pi / \pi^{n}\right)$ is equal to $\mathbb{Z}_{p}\left[\pi / \pi^{n}\right]$ and hence $H_{1}\left(\pi ; L\left(\pi / \pi^{n}\right)\right)=$ $\left(\pi^{n} /\left[\pi^{n}, \pi^{n}\right]\right) \otimes_{\mathbb{Z}} \mathbb{Z}_{p}$. It follows that $\pi^{(n+1)}$ is equal to $\pi^{n}$.

To prove that $\pi / \pi^{n+1} \cong \widehat{\pi} / \widehat{\pi}^{n+1}$, it suffices to show that $\pi^{n} / \pi^{n+1} \rightarrow \widehat{\pi}^{n} / \widehat{\pi}^{n+1}$ is an isomorphism, by applying the five lemma to the following diagram:

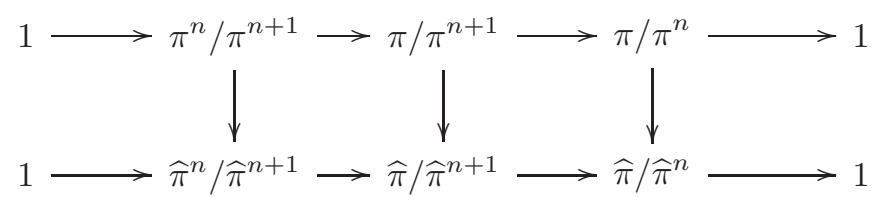

Since $\pi^{n} \rightarrow H_{1}\left(\pi ; L\left(\pi / \pi^{n}\right)\right)$ is surjective, $\pi^{n} / \pi^{n+1} \cong H_{1}\left(\pi ; L\left(\pi / \pi^{n}\right)\right)$, and similarly $\widehat{\pi}^{n} / \widehat{\pi}^{n+1} \cong H_{1}\left(\widehat{\pi} ; L\left(\pi / \pi^{n}\right)\right)$. The natural map $H_{1}\left(\pi ; L\left(\pi / \pi^{n}\right)\right) \rightarrow H_{1}\left(\widehat{\pi} ; L\left(\pi / \pi^{n}\right)\right)$ is the map $\alpha$ in the proof of Theorem 4.1, which was shown to be an isomorphism. This completes the proof.

Remark 4.4. Using Theorem 4.2, one can prove the result of Cochran and Harvey on the mod $p$ derived series [9, Corollary 4.3] using the newer tools of this paper. 


\section{ExAmPles OF COMPUTATION}

We will discuss examples illustrating interesting properties and elementary computational techniques of the local derived series. Some examples relate to later geometric applications.

The following lemma helps us compute lower terms of the Vogel-Cohn $R$-local derived series.

Lemma 5.1. Suppose $R$ is a subring of $\mathbb{Q}$ (and $\Gamma=\{e\}$ ). Then lower successive quotients of the Vogel-Cohn local derived series $\left\{\pi^{(n)}\right\}$ of a group $\pi$ are given as follows:

$$
\frac{\pi^{(n)}}{\pi^{(n+1)}}= \begin{cases}H_{1}(\pi ; \mathbb{Z}) / S \text {-torsion } & \text { for } n=0 \\ H_{1}\left(\pi ; \mathbb{Z}\left[\pi / \pi^{(1)}\right]\right) / \Sigma \text {-torsion } & \text { for } n=1\end{cases}
$$

where $S=\{s \in \mathbb{Z} \mid s$ is invertible in $R\}, \Sigma=\left\{x \in \mathbb{Z}\left[\pi / \pi^{(1)}\right] \mid \epsilon(x) \in S\right\}$, and $\epsilon: \mathbb{Z}\left[\pi / \pi^{(1)}\right] \rightarrow \mathbb{Z}$ is the augmentation map.

Using the lemma, $\pi^{(n+1)}$ can be computed as the kernel of the obvious map $\pi^{(n)} \rightarrow \pi^{(n)} / \pi^{(n+1)}$. (For example, for a group $\pi$ with a presentation, the ReidemeisterSchreier method may help in computing $\pi^{(n+1)}$ from $\pi^{(n)} / \pi^{(n+1)}$.)

Proof. For $n=0$, the conclusion is trivial. For $n=1$, note that $\pi^{(1)}$ is the kernel of

$$
\pi \longrightarrow H_{1}(\pi ; \mathbb{Z}) \longrightarrow H_{1}(\pi ; R)=H_{1}(\pi ; \mathbb{Z}) \otimes R .
$$

From this the conclusion follows, since the first map is surjective and the kernel of the second map is the $S$-torsion subgroup of $H_{1}(\pi ; \mathbb{Z})$.

For $n=2$, first note that since $\widehat{\pi} / \widehat{\pi}^{(1)}$ is abelian, the Cohn localization $\Lambda$ of the augmentation map $R\left[\widehat{\pi} / \widehat{\pi}^{(1)}\right] \rightarrow R$ is isomorphic to $\widehat{\Sigma}^{-1} R\left[\widehat{\pi} / \widehat{\pi}^{(1)}\right]$ where $\widehat{\Sigma}=\{x \in$ $\left.\mathbb{Z}\left[\widehat{\pi} / \widehat{\pi}^{(1)}\right] \mid \widehat{\epsilon}(x) \in S\right\}$ and $\widehat{\epsilon}$ is the augmentation $\mathbb{Z}\left[\widehat{\pi} / \widehat{\pi}^{(1)}\right] \rightarrow \mathbb{Z}$. Therefore $\pi^{(2)}$ is the kernel of

$$
\begin{aligned}
(*) \quad \pi^{(1)} \longrightarrow H_{1}\left(\pi ; \mathbb{Z}\left[\frac{\pi}{\pi^{(1)}}\right]\right) \stackrel{\alpha}{\longrightarrow} H_{1}\left(\pi ; \mathbb{Z}\left[\frac{\widehat{\pi}}{\widehat{\pi}^{(1)}}\right]\right) \\
\stackrel{\beta}{\longrightarrow} H_{1}(\pi ; \Lambda)=H_{1}\left(\pi ; \mathbb{Z}\left[\frac{\widehat{\pi}}{\widehat{\pi}^{(1)}}\right]\right) \underset{\mathbb{Z}\left[\frac{\widehat{\pi}^{(1)}}{\widehat{\pi}^{(1)}}\right]}{\otimes} \widehat{\Sigma}^{-1} \mathbb{Z}\left[\frac{\widehat{\pi}}{\widehat{\pi}^{(1)}}\right] .
\end{aligned}
$$

For the last equality, note that in this case, $\Lambda$ a commutative localization, and therefor flat.

Since $\pi / \pi^{(1)} \rightarrow \widehat{\pi} / \widehat{\pi}^{(1)}$ is injective, we view $\pi / \pi^{(1)}$ as a subgroup of $\widehat{\pi} / \widehat{\pi}^{(1)}$. Let $W$ be a set of coset representatives. As $\mathbb{Z}\left[\pi / \pi^{(1)}\right]$-modules, for each $g \in W$, $g \cdot \mathbb{Z}\left[\pi / \pi^{(1)}\right]$ is a submodule of $\mathbb{Z}\left[\widehat{\pi} / \widehat{\pi}^{(1)}\right]$ isomorphic to $\mathbb{Z}\left[\pi / \pi^{(1)}\right]$ and we have

$$
\mathbb{Z}\left[\widehat{\pi} / \widehat{\pi}^{(1)}\right]=\bigoplus_{g \in W} g \cdot \mathbb{Z}\left[\pi / \pi^{(1)}\right] .
$$

Also, we have

$$
(* * *) H_{1}\left(\pi ; \mathbb{Z}\left[\frac{\widehat{\pi}}{\widehat{\pi}^{(1)}}\right]\right) \cong H_{1}\left(\pi ; \mathbb{Z}\left[\frac{\pi}{\pi^{(1)}}\right]\right)_{\mathbb{Z}\left[\frac{\pi}{\pi^{(1)}}\right]}^{\otimes} \mathbb{Z}\left[\frac{\widehat{\pi}}{\widehat{\pi}^{(1)}}\right] \cong \bigoplus_{g \in W} g \cdot H_{1}\left(\pi ; \mathbb{Z}\left[\frac{\pi}{\pi^{(1)}}\right]\right) .
$$

In particular the map $\alpha$ in $(*)$ is injective and we can view $H_{1}\left(\pi ; \mathbb{Z}\left[\pi / \pi^{(1)}\right]\right)$ as a submodule of $H_{1}\left(\pi ; \mathbb{Z}\left[\widehat{\pi} / \widehat{\pi}^{(1)}\right]\right)$.

We assert that for any $x \in H_{1}\left(\pi ; \mathbb{Z}\left[\pi / \pi^{(1)}\right]\right), r \cdot x=0$ for some $r \in \widehat{\Sigma} \subset \mathbb{Z}\left[\widehat{\pi} / \widehat{\pi}^{(1)}\right]$ if and only if $t \cdot x=0$ for some $t \in \Sigma \subset \mathbb{Z}\left[\pi / \pi^{(1)}\right]$. The if part is clear. For the only if part, suppose $r \cdot x=0$ for some $r \in \widehat{\Sigma}$. We can write $r=\sum_{g \in W} g \cdot t_{g}$ with $t_{g} \in \mathbb{Z}\left[\pi / \pi^{(1)}\right]$ using $(* *)$. Since $x \in H_{1}\left(\pi ; \mathbb{Z}\left[\pi / \pi^{(1)}\right]\right)$, summands of $r \cdot x=$ $\sum_{g \in W} g \cdot\left(t_{g} \cdot x\right)$ are contained in distinct direct summands in $(* * *)$. It follows that 
$t_{g} \cdot x=0$ for each $g$. Let $t=\sum_{g \in W} t_{g} \in \mathbb{Z}\left[\pi / \pi^{(1)}\right]$. Obviously $t \cdot x=0$, and $t$ is in $\widehat{\Sigma}$ since $\epsilon(t)=\sum \epsilon\left(t_{i}\right)=\widehat{\epsilon}(r)$.

Note that for the map $\beta$ in $(*), \operatorname{Ker} \beta$ is equal to the $\widehat{\Sigma}$-torsion submodule of $H_{1}\left(\pi ; \mathbb{Z}\left[\widehat{\pi} / \widehat{\pi}^{(1)}\right]\right)$. Therefore, by the above assertion, Ker $\beta \alpha=($ pre-image of Ker $\beta$ ) is exactly the $\Sigma$-torsion submodule of $H_{1}\left(\pi ; \mathbb{Z}\left[\pi / \pi^{(1)}\right]\right)$. Since the first map in $(*)$ is surjective, from this it follows that $\pi^{(1)} / \pi^{(2)} \cong H_{1}\left(\pi ; \mathbb{Z}\left[\pi / \pi^{(1)}\right]\right) / \Sigma$-torsion.

Example 5.2. We will illustrate that in general the (integral) local derived series is distinct from the ordinary derived series and the Harvey series. Let $\pi=\mathbb{Z}_{p} \rtimes \mathbb{Z}_{2}$, where $p>1$ is an odd integer and $\mathbb{Z}_{p}$ is viewed as a $\mathbb{Z}\left[\mathbb{Z}_{2}\right]$-module via the negation action of $\mathbb{Z}_{2}$ on $\mathbb{Z}_{p}$. That is, denoting by $t$ the generator of $\mathbb{Z}_{2}, t^{-1} r t=-r$ in $\pi$ for $r \in \mathbb{Z}_{p}$.

First we will show that the ordinary derived series is as follows:

$$
\pi^{n}= \begin{cases}\pi & \text { for } n=0 \\ \mathbb{Z}_{p} & \text { for } n=1 \\ 0 & \text { for all } n \geq 2\end{cases}
$$

Proof. From the above definition of the $t$ action, it is easily seen that $\mathbb{Z}_{p}$ is in the kernel of the abelianization map and so $\pi / \pi^{1}=\mathbb{Z}_{2}, \pi^{1}=\mathbb{Z}_{p}$. Since $\pi^{1}$ is abelian, $\pi^{n}=0$ for $n \geq 2$.

On the other hand, for any finite group $\pi$ (including our case), it can be seen easily that the Harvey series $\left\{\pi_{H}^{(n)}\right\}$ is given as follows:

$$
\pi_{H}^{(n)}=\pi \quad \text { for all } n \text {. }
$$

Now we will show that the Vogel-Cohn local derived series of $\pi$ (for $R=\mathbb{Z}$ and $\Gamma=\{e\})$ is given by

$$
\pi^{(n)}= \begin{cases}\pi & \text { for } n=0 \\ \mathbb{Z}_{p} & \text { for } n \geq 1\end{cases}
$$

Proof. By Lemma 5.1, $\pi^{(0)}=\pi$ and $\pi / \pi^{(1)}=H_{1}(\pi ; \mathbb{Z})=\mathbb{Z}_{2}$ and thus $\pi^{(1)}=\pi^{1}=$ $\mathbb{Z}_{p}$. Also by Lemma [5.1] $\pi^{(1)} / \pi^{(2)}=H_{1}\left(\pi ; \mathbb{Z}\left[\mathbb{Z}_{2}\right]\right) / \Sigma$-torsion where

$$
\Sigma=\left\{a+b t \in \mathbb{Z}\left[\mathbb{Z}_{2}\right] \mid \epsilon(a+b t)=a+b=1\right\} .
$$

For $r \in \mathbb{Z}_{p}=\pi^{1} / \pi^{2}$ and $a+(1-a) t \in \Sigma$, the action is given by $(a+(1-a) t) \cdot r=$ $(a-(1-a)) r=(2 a-1) r$. In particular $r$ is annihilated by $a+(1-a) t \in \Sigma$ when $a=(p+1) / 2$. It follows that $H_{1}\left(\pi ; \mathbb{Z}\left[\mathbb{Z}_{2}\right]\right)$ is $\Sigma$-torsion. Therefore $\pi^{(2)}=\pi^{(1)}$.

Example 5.3. We will illustrate that even the rational (i.e., the largest) local derived series may be strictly smaller than the Harvey series. Our example is a group which is rationally Vogel-Cohn solvable but not Harvey solvable. This results from using the Cohn localization to compute the local derived series in place of the Ore localization used in the Harvey series, as our computation shows. Observe from this example that the Ore localization inverts excessively many ring elements, enlarging the size of the Harvey series.

Let $\pi=A \rtimes \mathbb{Z}$, where

$$
A=\frac{\mathbb{Z}\left[t, t^{-1}\right]}{\left\langle(t-1)^{2}\right\rangle}
$$

and a preferred generator, say $t \in \mathbb{Z}$, acts on $A$ by multiplication. Let $R$ be any subring of $\mathbb{Q}$ and $\pi^{(n)}$ be the $R$-coefficient Vogel-Cohn local derived series of $\pi$. We 
will show:

$$
\pi^{(n)}= \begin{cases}\pi & \text { for } n=0 \\ (t-1) A \cong \mathbb{Z} & \text { for } n=1 \\ 0 & \text { for } n \geq 2\end{cases}
$$

Proof. It can be seen that $[\pi, \pi]=(t-1) A \cong \mathbb{Z}$ and $H_{1}(\pi ; \mathbb{Z})=\pi /[\pi, \pi] \cong \mathbb{Z} \times \mathbb{Z}$ is a torsion free abelian group generated by (the cosets of) $1 \in A$ and $t \in \mathbb{Z}$. Therefore, by Lemma $5.1 \pi / \pi^{(1)}=H_{1}(\pi ; \mathbb{Z})=\mathbb{Z} \times \mathbb{Z}$ and so $\pi^{(1)}=[\pi, \pi]$. Since $\pi^{(1)}$ is abelian, $\pi^{(1)}=H_{1}(\pi ; \mathbb{Z}[\mathbb{Z} \times \mathbb{Z}])$. It can be verified easily that the $\mathbb{Z} \times \mathbb{Z}$-action on $H_{1}(\pi ; \mathbb{Z}[\mathbb{Z} \times \mathbb{Z}]) \cong(t-1) A$ is trivial. From this it follows that no nontrivial element in $H_{1}(\pi ; \mathbb{Z}[\mathbb{Z} \times \mathbb{Z}])$ is $\Sigma$-torsion, where $\Sigma$ is as in Lemma [5.1 Therefore $0=\pi^{(2)}=\pi^{(3)}=\cdots$ by Lemma 5.1 .

Now we will compute the Harvey series to show

$$
\pi_{H}^{(n)}= \begin{cases}\pi & \text { for } n=0 \\ (t-1) A \cong \mathbb{Z} & \text { for } n \geq 1\end{cases}
$$

Proof. Since $H_{1}(\pi ; \mathbb{Z})$ is torsion free, $\pi_{H}^{(1)}=[\pi, \pi]=(t-1) A \cong \mathbb{Z}$ and $\pi / \pi_{H}^{(1)}=$ $H_{1}(\pi ; \mathbb{Z})=\mathbb{Z} \times \mathbb{Z}$. To compute $\pi_{H}^{(2)}$, recall that $\pi_{H}^{(2)}$ is the kernel of

$$
\pi_{H}^{(1)}=H_{1}(\pi ; \mathbb{Z}[\mathbb{Z} \times \mathbb{Z}]) \longrightarrow H_{1}(\pi ; \mathbb{Z}[\mathbb{Z} \times \mathbb{Z}])_{\mathbb{Z}[\mathbb{Z} \times \mathbb{Z}]}^{\otimes} \mathcal{K}(\mathbb{Z} \times \mathbb{Z})
$$

where $\mathcal{K}(\mathbb{Z} \times \mathbb{Z})$ denotes the Ore localization of $\mathbb{Z}[\mathbb{Z} \times \mathbb{Z}]$. Since the action of $\mathbb{Z} \times \mathbb{Z}$ is trivial on $\pi_{H}^{(1)}$, every element of $\pi_{H}^{(1)}$ is annihilated by any $r \neq 0 \in \mathbb{Z}[\mathbb{Z} \times \mathbb{Z}]$ which auguments to zero (i.e., $\epsilon(r)=1$ ). It follows that the above homomorphism is a zero map. Therefore we have $\pi_{H}^{(1)}=\pi_{H}^{(2)}=\cdots$.

We remark that the elements $r$ used above (such that $\epsilon(r)=0$ ) are inverted in the Ore localization but not inverted in the Cohn localization.

Example 5.4. For a finitely generated free group $F$, all the concerned commutatortype series agree; it is known that $F^{n}=F_{H}^{(n)}$ for any $n$ [10, and so by Theorem 4.1] it follows that $F^{n}=F^{\{n\}}=F^{(n)}=F_{H}^{(n)}$ for any $n$.

Example 5.5. In what follows we describe the Vogel-Cohn local derived series of all the fundamental groups of 3-dimensional linear spherical space forms, that is, all finite groups acting on $S^{3}$ linearly without fixed points (see Milnor 25]). Since Lemma 5.1 (together with the Reidemeister-Schreier method if necessary) applies effectively, we omit details of the computation. Let $R$ be a fixed subring of $\mathbb{Q}$.

(1) $\pi=Q_{8 n}=\left\langle x, y \mid y^{2 n}=x^{2}=(x y)^{2}\right\rangle$ with $n \geq 1$ : write $n=2^{r} \cdot n_{0}$ with $n_{0}$ odd. If $1 / 2 \notin R$, then

$$
\frac{\pi^{(n)}}{\pi^{(n+1)}} \cong \begin{cases}\mathbb{Z}_{2} \times \mathbb{Z}_{2} \text { generated by } x \text { and } y, & n=0, \\ \mathbb{Z}_{2^{r+1}} \text { generated by } y^{2}, & n=1,\end{cases}
$$

and

$$
\pi^{(n)}= \begin{cases}\left\langle y^{2} \mid\left(y^{2}\right)^{2 n}=1\right\rangle \cong \mathbb{Z}_{2 n}, & n=1, \\ \left\langle y^{2^{r+2}} \mid\left(y^{2^{r+2}}\right)^{n_{0}}=1\right\rangle \cong \mathbb{Z}_{n_{0}} \text { generated by } y^{2^{r+2}}, & n=2 .\end{cases}
$$

If $1 / 2 \in R$, then $\pi^{(n)}=\pi$ for all $n$.

(2) $\pi=P_{48}=\left\langle x, y \mid x^{2}=(x y)^{3}=y^{4}, x^{4}=1\right\rangle$ : if $1 / 2 \notin R$, then

$$
\frac{\pi^{(n)}}{\pi^{(n+1)}} \cong \begin{cases}\mathbb{Z}_{2} \text { generated by } x, & n=0, \\ 0 & n \geq 1,\end{cases}
$$


and

$$
\pi^{(n)}=\left\langle u, v \mid u^{2}=v^{3}, u v^{2} u v^{2} u=v, u^{4}=1\right\rangle
$$

for $n \geq 1$, where $u=y^{2}, v=x y$. If $1 / 2 \in R$, then $\pi^{(n)}=\pi$ for all $n$.

(3) $\pi=P_{120}=\left\langle x, y \mid x^{2}=(x y)^{3}=y^{5}, x^{4}=1\right\rangle: \pi$ is perfect and so $\pi^{(n)}=\pi$ for any $n$ and any $R$.

(4) $\pi=D_{2^{k}(2 n+1)}=\left\langle x, y \mid x^{2 k}=1, y^{2 n+1}=1, x y x^{-1}=y^{-1}\right\rangle$ : if $1 / 2 \notin R$, then

and for $n \geq 1$,

$$
\frac{\pi^{(n)}}{\pi^{(n+1)}} \cong \begin{cases}\mathbb{Z}_{2^{k}} \text { generated by } x, & n=0 \\ 0 & n \geq 1\end{cases}
$$

$$
\pi^{(n)}=\left\langle y \mid y^{2 n+1}=1\right\rangle \cong \mathbb{Z}_{2 n+1} .
$$

If $1 / 2 \in R$, then $\pi^{(n)}=\pi$ for all $n$.

(5) $\pi=P_{8 \cdot 3^{k}}^{\prime}=\left\langle x, y, z \mid x^{2}=(x y)^{2}=y^{2}, z^{-1} x z=y, z^{-1} y z=x y, z^{3^{k}}=1\right\rangle$ : if $1 / 3 \notin R$, then

$$
\frac{\pi^{(n)}}{\pi^{(n+1)}} \cong \begin{cases}\mathbb{Z}_{3^{k}} \text { generated by } z, & n=0 \\ 0 & n \geq 1\end{cases}
$$

and for $n \geq 1$,

$$
\pi^{(n)}=\left\langle x, y \mid x y x^{-1}=y^{-1}, x^{2}=y^{2}\right\rangle .
$$

If $1 / 3 \in R$, then $\pi^{(n)}=\pi$ for all $n$.

(6) $\pi=\mathbb{Z}_{d} \times G$ where $G$ is either trivial or any group listed above: first consider $\pi=\mathbb{Z}_{d}$. If $1 / d \notin R$, then $\mathbb{Z}_{d}^{(n)}=0$ for any $n \geq 1$. If $1 / d \in R$, then $\mathbb{Z}_{d}^{(n)}=\mathbb{Z}_{d}$ for any $n$. For the case of a product $\pi=\mathbb{Z}_{d} \times G$, the following lemma applies.

Lemma 5.6. For the direct product $\pi=G \times H$ of two groups $G$ and $H, \pi^{(n)}=$ $G^{(n)} \times H^{(n)}$ for any $n$.

We remark that this product formula holds for the local derived series determined by any homology localization triple.

Proof. We use an induction on $n$. For $n=0$, the conclusion is trivial. The conclusion for a limit ordinal follows easily by taking limits. To consider the case of a successor ordinal, suppose $\pi^{(n)}=G^{(n)} \times H^{(n)}$. Consider the following diagram:

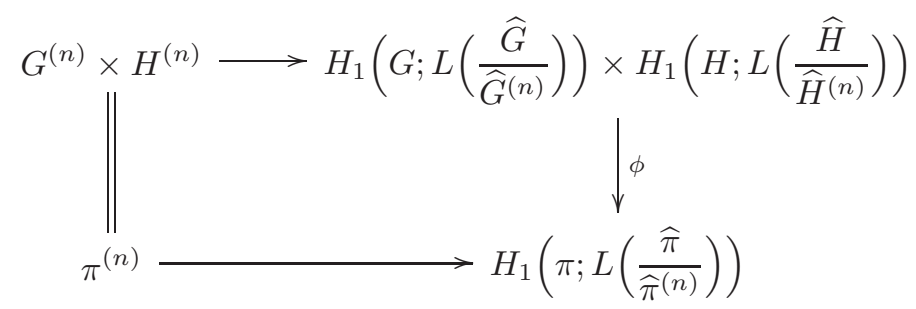

where the map $\phi$ is the product of the maps induced by the inclusions of $G$ and $H$ into $\pi$. Note that there are induced maps on the homology coefficients by the functoriality of $(-) /(-)^{(n)}$ and $L$. By the functoriality of the group localization, the projection induces $\widehat{\pi} \rightarrow \widehat{G}$ which is a left inverse of $\widehat{G} \rightarrow \widehat{\pi}$. Therefore there is an induced map $\alpha: H_{1}\left(\pi ; L\left(\widehat{\pi} / \widehat{\pi}^{(n)}\right)\right) \rightarrow H_{1}\left(G ; L\left(\widehat{G} / \widehat{G}^{(n)}\right)\right)$. A similar argument applied to $H$ gives a map $\beta: H_{1}\left(\pi ; L\left(\widehat{\pi} / \widehat{\pi}^{(n)}\right)\right) \rightarrow H_{1}\left(H ; L\left(\widehat{H} / \widehat{H}^{(n)}\right)\right)$. Then the map $(\alpha, \beta)$ on $H_{1}\left(\pi ; L\left(\widehat{\pi} / \widehat{\pi}^{(n)}\right)\right)$ is a left inverse of the map $\phi$. Therefore $\phi$ is injective. From this it follows that $\pi^{(n+1)}=G^{(n+1)} \times H^{(n+1)}$ by a diagram chase. 
We finish this section by emphasizing that $\pi / \pi^{(n)}$ may have nontrivial torsion elements (e.g., see Example 5.5). In contrast to this, the Harvey series quotient $\pi / \pi_{H}^{(n)}$ is always torsion-free. The existence of torsion elements enables us to make some interesting applications of the local derived series to homology cobordism of manifolds, as discussed in later sections.

\section{6. $L^{2}$-Dimensional LOCAL PROPERTY OF VON NEUMANN GROUP ALGEBRAS}

In this section we will prove that the von Neumann group algebra of a group ring behaves much like a Cohn local module over the underlying group ring for an appropriate collection of groups. This " $L^{2}$-dimensional Cohn local property" of the von Neumann group algebra $\mathcal{N} G$ (Theorem 6.6), has significant geometric applications related to homological properties. The essential new contribution is that it holds even for a class of groups $G$ that may have torsion (as well as infinite order elements). We start by defining the notion of an $L^{2}$-equivalence.

6.1. $L^{2}$-dimension and $L^{2}$-equivalences. Suppose $G$ is a countable group with von Neumann group algebra $\mathcal{N} G$. Following Lück's book [24, Chapter 6], the $L^{2}$ dimension function

$$
\operatorname{dim}^{(2)}:\{\mathcal{N} G \text {-modules }\} \longrightarrow[0, \infty]=[0, \infty) \cup\{\infty\}
$$

is defined as follows. For a finitely generated projective $\mathcal{N} G$-module $P$, $\operatorname{dim}^{(2)} P$ is defined to be the von Neumann trace of a square matrix over $\mathcal{N} G$ whose row space is isomorphic to $P$. For an arbitrary module $M$ over $\mathcal{N} G$, $\operatorname{dim}^{(2)} M=\sup \left\{\operatorname{dim}^{(2)} P \mid\right.$ $P$ is a finitely generated projective submodule of $M$ \}.

In this paper we will frequently use the following basic properties of the $L^{2}$ dimension function:

(1) $\operatorname{dim}^{(2)} \mathcal{N} G=1$ and $\operatorname{dim}^{(2)} 0=0$.

(2) If $0 \rightarrow M^{\prime} \rightarrow M \rightarrow M^{\prime \prime} \rightarrow 0$ is exact, then $\operatorname{dim}^{(2)} M=\operatorname{dim}^{(2)} M^{\prime}+$ $\operatorname{dim}^{(2)} M^{\prime \prime}$. In particular, if $N$ is either a submodule or a homomorphic image of $M$, then $\operatorname{dim}^{(2)} N \leq \operatorname{dim}^{(2)} M$.

For proofs, see [24, Chapter 6]. Here we adopt the usual convention $\infty+d=\infty$ for any $d \in[0, \infty]$.

Definition 6.1. An $\mathcal{N} G$-module homomorphism $f: M \rightarrow N$ is said to be an $L^{2}$ equivalence if $\operatorname{dim}^{(2)} \operatorname{Ker} f=0=\operatorname{dim}^{(2)}$ Coker $f$.

\section{Remark 6.2.}

(1) If $f: M \rightarrow N$ is an $L^{2}$-equivalence, $\operatorname{dim}^{(2)} M=\operatorname{dim}^{(2)} N=\operatorname{dim}{ }^{(2)} \operatorname{Im} f$.

(2) If $\operatorname{dim}^{(2)} M=\operatorname{dim}^{(2)} N<\infty$, then we have $\operatorname{dim}^{(2)} \operatorname{Ker} f=\operatorname{dim}^{(2)}$ Coker $f$ for any $f: M \rightarrow N$. In particular in this case $f$ is an $L^{2}$-equivalence if and only if either Ker $f$ or Coker $f$ has $\operatorname{dim}^{(2)}=0$.

A group $G$ is called amenable if $\mathcal{N} G \otimes_{\mathbb{C} G} \mathbb{C}$ is nonzero. For other equivalent definitions (which are more often used in the literature) and related discussions, the reader is referred to Paterson's book [26] and Lück's book [24, p. 256]. For our purpose, the following fact on amenable groups will play an essential role.

Theorem 6.3 (Lück [24, Theorem 6.37]). Suppose $G$ is an amenable group. Then $\mathcal{N} G$ is $L^{2}$-dimension flat over $\mathbb{C} G$, that is, $\operatorname{dim}^{(2)} \operatorname{Tor}_{p}^{\mathbb{C} G}(M, \mathcal{N} G)=0$ for any $\mathbb{C} G$-module $M$ and for any $p>0$.

Lemma 6.4. Suppose $G$ is amenable. If $M$ and $N$ are free $\mathbb{C} G$-modules with the same finite rank and $f: M \rightarrow N$ is an injection, then $f \otimes 1_{\mathcal{N} G}: M \otimes_{\mathbb{C} G} \mathcal{N} G \rightarrow$ $N \otimes_{\mathbb{C} G} \mathcal{N} G$ is an $L^{2}$-equivalence. 
Proof. Let $C=$ Coker $f$. From $0 \rightarrow M \rightarrow N \rightarrow C \rightarrow 0$ we obtain

$$
0 \longrightarrow \operatorname{Tor}_{1}^{\mathbb{C} G}(C, \mathcal{N} G) \longrightarrow M \underset{\mathbb{C} G}{\otimes} \mathcal{N} G \longrightarrow N \underset{\mathbb{C} G}{\otimes} \mathcal{N} G \longrightarrow C \underset{\mathbb{C} G}{\otimes} \mathcal{N} G \longrightarrow 0 .
$$

By Theorem 6.3 $\operatorname{dim}^{(2)} \operatorname{Tor}_{1}^{\mathbb{C} G}(C, \mathcal{N} G)=0$. Since $M \otimes_{\mathbb{C} G} \mathcal{N} G$ and $N \otimes_{\mathbb{C} G} \mathcal{N} G$ are finitely generated free $\mathcal{N} G$-modules with the same rank, they have the same finite $\operatorname{dim}^{(2)}$. It follows that $f \otimes 1_{\mathcal{N} G}$ is an $L^{2}$-equivalence.

The following is an $L^{2}$-equivalence analogue of the observation that a chain map which is an isomorphism induces an isomorphism on the homology.

Lemma 6.5. Suppose $\phi_{*}: C_{*} \rightarrow C_{*}$ is a chain map of a chain complex $C_{*}$ over $\mathcal{N} G$. If $\operatorname{dim}^{(2)} C_{i}<\infty$ and $\phi_{i}: C_{i} \rightarrow C_{i}$ is an $L^{2}$-equivalence for some $i$, then the induced $\operatorname{map}\left(\phi_{i}\right)_{*}: H_{i}\left(C_{*}\right) \rightarrow H_{i}\left(C_{*}\right)$ is an $L^{2}$-equivalence.

Proof. Let $Z_{i}, B_{i} \subset C_{i}$ be the submodules of cycles and boundaries, respectively. Consider $\left.\phi_{i}\right|_{Z_{i}}: Z_{i} \rightarrow Z_{i}$. Since Ker $\left.\phi_{i}\right|_{Z_{i}}=Z_{i} \cap \operatorname{Ker} \phi_{i}$ is a submodule of Ker $\phi_{i}$ and $\operatorname{dim}^{(2)} \operatorname{Ker} \phi_{i}=0$, we have $\left.\operatorname{dim}^{(2)} \operatorname{Ker} \phi_{i}\right|_{Z_{i}}=0$. Since $\operatorname{dim}^{(2)} Z_{i} \leq \operatorname{dim}^{(2)} C_{i}<\infty$, it follows that $\left.\phi_{i}\right|_{Z_{i}}$ is an $L^{2}$-equivalence.

$$
\operatorname{Coker}\left\{\left(\phi_{i}\right)_{*}: Z_{i} / B_{i} \longrightarrow Z_{i} / B_{i}\right\}=Z_{i} /\left(B_{i}+\phi_{i}\left(Z_{i}\right)\right)
$$

is a homeomorphic image of Coker $\left.\phi_{i}\right|_{Z_{i}}=Z_{i} / \phi_{i}\left(Z_{i}\right)$ and therefore

$$
\operatorname{dim}^{(2)} \operatorname{Coker}\left(\phi_{i}\right)_{*} \leq\left.\operatorname{dim}^{(2)} \operatorname{Coker} \phi_{i}\right|_{Z_{i}}=0 .
$$

Since $\operatorname{dim}^{(2)} Z_{i} / B_{i} \leq \operatorname{dim}^{(2)} C_{i}<\infty$, it follows that $\left(\phi_{i}\right)_{*}$ is an $L^{2}$-equivalence.

6.2. $\mathcal{N} G$-homology and Strebel's class $D(R)$. Suppose $R$ is a commutative ring. We always assume that a commutative $\operatorname{ring} R$ has unity and $1 \neq 0$ in $R$, i.e., the natural map $\mathbb{Z} \rightarrow R$ is nonzero. In [28], Strebel defined and studied the class $D(R)$ of groups $G$ with the following property: whenever $f: M \rightarrow N$ is a homomorphism between projective $R G$-modules such that $f \otimes 1_{R}: M \otimes_{R G} R \rightarrow$ $N \otimes_{R G} R$ is injective, $f$ itself is injective. (Here $R$ is viewed as an $R G$-module with trivial $G$-action.)

In the following theorem, we relate the class $D(R)$ with the $L^{2}$-dimension of $\mathcal{N} G$-homology modules.

Theorem 6.6. Suppose $R$ is a commutative ring, $G$ is an amenable group, and $G \rightarrow \Gamma$ is a group homomorphism with kernel in $D(R)$. Suppose $C_{*}$ is a bounded below chain complex over $\mathbb{Z} G$ such that $C_{i}$ is finitely generated and free as a $\mathbb{Z} G$ module for $i \leq n$. If $H_{i}\left(C_{*} \otimes_{\mathbb{Z} G} R \Gamma\right)=0$ for $i \leq n$, then $\operatorname{dim}^{(2)} H_{i}\left(C_{*} \otimes_{\mathbb{Z} G} \mathcal{N} G\right)=0$ for $i \leq n$.

\section{Remark 6.7.}

(1) Theorem6.6 will be useful in proving our results on $R \Gamma$-coefficient homology cobordism. Our primary examples applying this theorem will use the rings $R=\mathbb{Z}_{p}$ and $R=\mathbb{Q}$ in Theorem 6.6. One can consider subrings $R \subset \mathbb{Q}$ (including $\mathbb{Z}$ and $\mathbb{Z}_{(p)}$ ), but this serves no purpose since in this case, $D(R)=$ $D(\mathbb{Q})$. On the other hand, the classes $D(\mathbb{Q})$ and $D\left(\mathbb{Z}_{p}\right)$ are distinct. For example, a finite $p$-group is in $D\left(\mathbb{Z}_{p}\right)$ but not in $D(\mathbb{Q})$. See also Lemma 6.8.

(2) It is well known that a similar result to Theorem 6.6 holds for the Cohn localization of $\mathbb{Z} G \rightarrow \mathbb{Z} \Gamma$. (See, for instance, [30.) In this sense, we can interpret the conclusion of Theorem 6.6 as follows: the von Neumann group ring $\mathcal{N} G$ is an $L^{2}$-dimension Cohn local $\mathbb{Z} G$-module.

Proof of Theorem 6.6. We will show that the zero map of $H_{i}\left(C_{*} \otimes_{\mathbb{Z} G} \mathcal{N} G\right)$ into itself is an $L^{2}$-equivalence. From this the desired conclusion follows immediately. 
Our argument is similar to Vogel's argument used in [30] but requires Lemma 6.4 at a crucial point. Since $H_{i}\left(C_{*} \otimes_{\mathbb{Z} G} R \Gamma\right)=0$ for $i \leq n$, there is a partial chain homotopy

$$
s_{i}: C_{i} \underset{\mathbb{Z} G}{\otimes} R \Gamma \longrightarrow C_{i+1} \underset{\mathbb{Z} G}{\otimes} R \Gamma \quad(i \leq n)
$$

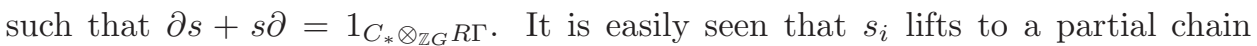
homotopy $D_{i}: C_{i} \rightarrow C_{i+1}(i \leq n)$ such that $D \otimes_{\mathbb{Z} G} 1_{R \Gamma}=s$. Let $N=\operatorname{Ker}\{G \rightarrow \Gamma\}$. For the partial chain map $\phi=\partial D+D \partial$ on $C_{*}$,

$$
\left(\phi_{i} \underset{\mathbb{Z}}{\otimes} 1_{R}\right) \underset{R N}{\otimes} 1_{R}:\left(C_{i} \underset{\mathbb{Z}}{\otimes} R\right) \underset{R N}{\otimes} R \longrightarrow\left(C_{i} \underset{\mathbb{Z}}{\otimes} R\right) \underset{R N}{\otimes} R
$$

can be identified with

$$
\phi_{i} \underset{\mathbb{Z} G}{\otimes} 1_{R \Gamma}: C_{i} \underset{\mathbb{Z} G}{\otimes} R \Gamma \longrightarrow C_{i} \underset{\mathbb{Z} G}{\otimes} R \Gamma
$$

which is the identity map $(i \leq n)$. Therefore, since $N$ is in $D(R)$,

$$
\phi_{i} \underset{\mathbb{Z}}{\otimes} 1_{R}: C_{i} \underset{\mathbb{Z}}{\otimes} R \longrightarrow C_{i} \underset{\mathbb{Z}}{\otimes} R
$$

is injective. Note that for any (possibly infinite) index set $A$, a homomorphism $\mathbb{Z}^{A} \rightarrow \mathbb{Z}^{A}$ is injective if the induced map $R^{A} \rightarrow R^{A}$ is injective. From this it follows that $\phi_{i}: C_{i} \rightarrow C_{i}$ is injective. Now, by Lemma 6.4,

$$
\phi_{i} \otimes 1_{\mathcal{N} G}: C_{i} \underset{\mathbb{Z} G}{\otimes} \mathcal{N} G \longrightarrow C_{i} \underset{\mathbb{Z} G}{\otimes} \mathcal{N} G
$$

is an $L^{2}$-equivalence. Therefore, by Lemma 6.5 it follows that $\phi_{i}$ induces an $L^{2}$ equivalence

$$
\phi_{*}: H_{i}\left(C_{*} \underset{\mathbb{Z} G}{\otimes} \mathcal{N} G\right) \longrightarrow H_{i}\left(C_{*} \underset{\mathbb{Z} G}{\otimes} \mathcal{N} G\right) .
$$

Because of $D_{*}$, the induced map $\phi_{*}$ is zero for $i \leq n$.

Note that if $\Gamma$ is trivial in Theorem 6.6. or more generally if $\Gamma$ is amenable, then $G$ is amenable if and only if $\operatorname{Ker}\{G \rightarrow \Gamma\}$ is amenable, since the class of amenable groups is closed under extensions and taking subgroups and quotients. Therefore the hypothesis of Theorem 6.6 leads us to consider the class of groups which are amenable and in $D(R)$. We list some known cases (e.g., see [26], 24], and [28]).

(1) Poly-torsion-free-abelian (PTFA) groups are amenable and in $D(R)$ for any ring $R$.

(2) Finite $p$-groups are amenable and in $D\left(\mathbb{Z}_{p}\right)$.

(3) A direct limit of amenable groups (resp. groups in $D(R)$ ) is amenable (resp. in $D(R))$.

(4) If $G$ admits a subnormal series

$$
G=G_{0} \supset G_{1} \supset \cdots \supset G_{n}=\{e\}
$$

such that each $G_{i} / G_{i+1}$ is amenable (resp. in $D(R)$ ), then $G$ is amenable (resp. in $D(R)$ ).

The following consequence will be used later. We say that a group $G$ has no torsion coprime to $p$ if the order of any finite order element in $G$ is a power of $p$. (Note that $G$ may have infinite order elements and may be infinitely generated.)

Lemma 6.8. Suppose $G$ is a group admitting a subnormal series

$$
G=G_{0} \supset G_{1} \supset \cdots \supset G_{n}=\{e\}
$$

whose quotients $G_{i} / G_{i+1}$ are abelian.

(1) If every $G_{i} / G_{i+1}$ has no torsion coprime to $p$, then $G$ is amenable and in $D\left(\mathbb{Z}_{p}\right)$.

(2) If every $G_{i} / G_{i+1}$ is torsion free, then $G$ is amenable and in $D(R)$ for any commutative ring $R$. 
Proof. (1) It suffices to show that $G_{i} / G_{i+1}$ is amenable and in $D(R)$. Note that $G_{i} / G_{i+1}$ is the direct limit of its finitely generated subgroups. Since $G_{i} / G_{i+1}$ is abelian and has no torsion coprime to $p$, each finitely generated subgroup $H$ of $G_{i} / G_{i+1}$ is a direct sum of abelian $p$-groups and free abelian groups. Therefore $H$ is amenable and in $D\left(\mathbb{Z}_{p}\right)$. It follows that $G_{i} / G_{i+1}$ is amenable and in $D\left(\mathbb{Z}_{p}\right)$. (Amenability of $G_{i} / G_{i+1}$ also follows immediately from that it is abelian.)

(2) In this case, $G_{i} / G_{i+1}$ is torsion-free and so is a direct limit of finitely generated free abelian groups. It follows that $G$ is amenable and in $D(R)$.

\section{7. $L^{2}$-INVARIANTS AND HOMOLOGY COBORDISM}

For a CW-complex $X$ and a group homomorphism $\phi: \pi_{1}(X) \rightarrow G$, we define the $L^{2}$-Betti number by $b_{i}^{(2)}(X, \phi)=\operatorname{dim}^{(2)} H_{i}(X ; \mathcal{N} G)$. When $X=M$ is a $(4 k-1)$ dimensional manifold, we denote by $\rho^{(2)}(M, \phi)$ the $L^{2}$-signature defect of a bounding $4 k$-manifold over $G$.

For the reader's convenience, we sketch the definition of $\rho^{(2)}(M, \phi)$. It is known that there exist a compact $4 k$-manifold $W$ with $\partial W=M$, an injection $G \rightarrow H$ of groups, and a homomorphism $\psi: \pi_{1}(W) \rightarrow H$ such that the following diagram commute:

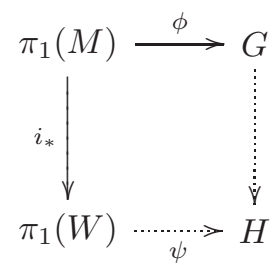

Applying the spectral theory to the $\mathcal{N} H$-coefficient intersection form

$$
H_{2 k}(W ; \mathcal{N} H) \times H_{2 k}(W ; \mathcal{N} H) \longrightarrow \mathcal{N} H
$$

$H_{2 k}(W ; \mathcal{N} H)$ can be written as an orthogonal sum $V_{+} \oplus V_{-} \oplus V_{0}$ where the intersection form on $V_{+}, V_{-}$, and $V_{0}$ are positive definite, negative definite, and zero, respectively. The $L^{2}$-signature of $(W, \psi)$ is defined to be

$$
\operatorname{sign}^{(2)}(W, \psi)=\operatorname{dim}^{(2)} V_{+}-\operatorname{dim}^{(2)} V_{-} .
$$

Now the $\rho$-invariant $\rho^{(2)}(M, \phi)$ is defined to be the $L^{2}$-signature defect of $W$, namely

$$
\rho^{(2)}(M, \phi)=\operatorname{sign}^{(2)}(W, \psi)-\operatorname{sign}(W)
$$

where $\operatorname{sign}(W)$ is the ordinary signature of $W$. It is well known that $\rho^{(2)}(M, \phi)$ is a well-defined real-valued invariant of $(M, \phi)$, which agrees with the Cheeger-Gromov invariant. For more details and related discussions, the reader may be referred to, for example, Cochran-Orr-Teichner [14, Chang-Weinberger 8, Lück [24, Harvey [19, and Cha 7 .

We also need the induction property: if $\phi: \pi_{1}(M) \rightarrow G$ is a homomorphism and $f: G \rightarrow H$ is an injection, then $\rho^{(2)}(M, \phi)=\rho^{(2)}(M, f \phi)$.

We say that a (connected) complex $X$ is over a group $\Gamma$ if $X$ is endowed with a homomorphism $\pi_{1}(X) \rightarrow \Gamma$. For two closed manifolds $M$ and $M^{\prime}$ over $\Gamma$ and a commutative ring $R$, a bordism $W$ over $\Gamma$ between $M$ and $M^{\prime}$ is called an $R \Gamma$ homology cobordism if the inclusions of $M$ and $M^{\prime}$ into $W$ induce isomorphisms on $H_{*}(-; R \Gamma)$. If such $W$ exists, $M$ and $M^{\prime}$ are said to be $R \Gamma$-homology cobordant.

Theorem 7.1. Suppose $R$ is a commutative ring, $G$ is an amenable group, and $G \rightarrow \Gamma$ is a homomorphism with kernel in $D(R)$. Suppose $W$ is an $R \Gamma$-homology cobordism between closed manifolds $M$ and $M^{\prime}$ over $\Gamma$. If $\phi: \pi_{1}(M) \rightarrow G$ and $\phi^{\prime}: \pi_{1}\left(M^{\prime}\right) \rightarrow G$ are restrictions of a homomorphism $\psi: \pi_{1}(W) \rightarrow G$, then the following hold: 
(1) $b_{i}^{(2)}(M, \phi)=b_{i}^{(2)}\left(M^{\prime}, \phi^{\prime}\right)$ for all $i$.

(2) When $M$ and $M^{\prime}$ are $(4 k-1)$-dimensional, $\rho^{(2)}(M, \phi)=\rho^{(2)}\left(M^{\prime}, \phi^{\prime}\right)$.

Note that $W$ is a $\mathbb{Z}_{p} \Gamma$-homology cobordism if and only if $W$ is a $\mathbb{Z}_{(p)} \Gamma$-homology cobordism. Using this fact we can combine the $\mathbb{Z}_{(p)}$-coefficient Vogel-Cohn local derived series with the $L^{2}$-signatures associated to amenable groups in $D\left(\mathbb{Z}_{p}\right)$. A precise statement is as follows.

Theorem 7.2. Let $R$ be either $\mathbb{Z}_{p}, \mathbb{Z}_{(p)}$, or $\mathbb{Q}$. For a closed manifold $M$ over an amenable group $\Gamma$, view $\pi=\pi_{1}(M)$ as a group over $\Gamma$ and denote the $R$ coefficient Vogel-Cohn local derived series of $\pi$ over $\Gamma$ by $\left\{\pi^{(n)}\right\}$. For the canonical map $\phi_{n}: \pi \rightarrow \pi / \pi^{(n)}, b^{(2)}\left(M, \phi_{n}\right)$ and $\rho^{(2)}\left(M, \phi_{n}\right)$ are $R \Gamma$-homology cobordism invariants of $M$ for any $n<\infty$. In particular, when $\Gamma$ is trivial, $b^{(2)}\left(M, \phi_{n}\right)$ and $\rho^{(2)}\left(M, \phi_{n}\right)$ are always $R$-homology cobordism invariants.

We remark that in many cases the group $\pi_{1}(M) / \pi_{1}(M)^{(n)}$ may have torsion elements. (For instance, see our computation for spherical space forms: Example 5.5.) Prior to this work, only $L^{2}$-signatures associated to poly-torsion-free-abelian groups have been known to be useful in studying homology cobordism. Theorem 7.2 is the first result on the homology cobordism invariance of $L^{2}$-signatures associated to non-torsion-free groups.

Proof of Theorem 7.1. Since $H_{i}(W, M ; R \Gamma)=0$, applying Theorem 6.6 to the chain complex $C_{*}=C_{*}(W, M ; \mathbb{Z} G)$ we obtain $\operatorname{dim}^{(2)} H_{i}(W, M ; \mathcal{N} G)=0$ for all $i$. From the $\mathcal{N} G$-coefficient homology long exact sequence for $(W, M)$, it follows that the map $H_{i}(M ; \mathcal{N} G) \rightarrow H_{i}(W ; \mathcal{N} G)$ is an $L^{2}$-equivalence. The same argument applies to $M^{\prime}$. Therefore $b_{i}^{(2)}(M, \phi)=b_{i}^{(2)}(W, \psi)=b_{i}^{(2)}\left(M^{\prime}, \phi^{\prime}\right)$.

For $(2)$, note that $\rho^{(2)}(M, \phi)-\rho^{(2)}\left(M^{\prime}, \phi^{\prime}\right)$ is the difference between the $L^{2}$ signature of the $\mathcal{N} G$-intersection form of $W$ and the signature of the ordinary (untwisted) intersection form of $W$. We will show that both signatures are zero.

The non-singular part of the $\mathcal{N} G$-intersection form is supported by the cokernel of $H_{2 k}(M ; \mathcal{N} G) \rightarrow H_{2 k}(W ; \mathcal{N} G)$ which is contained in $H_{2 k}(W, M ; \mathcal{N} G)$. Since $\operatorname{dim}^{(2)} H_{2 k}(W, M ; \mathcal{N} G)$ is zero, the $L^{2}$-signature of $W$ is zero. Similarly, the ordinary signature of $W$ is zero, since $H_{2 k}(W, M ; \mathbb{Q})=0$.

Proof of Theorem 7.2. We first consider the case $R=\mathbb{Z}_{(p)}$. Suppose $W$ is a $R \Gamma$ homology cobordism between $M$ and $M^{\prime}$. Let $G=\pi_{1}(W) / \pi_{1}(W)^{(n)}$, and let $\phi: \pi_{1}(M) \rightarrow \pi_{1}(W) \rightarrow G$ and $\phi^{\prime}: \pi_{1}\left(M^{\prime}\right) \rightarrow \pi_{1}(W) \rightarrow G$ be the compositions. By the injectivity theorem, $\pi_{1}(M) / \pi_{1}(M)^{(n)}$ injects into $G$. Therefore by the induction property, $\rho^{(2)}\left(M, \phi_{n}\right)$ is equal to $\rho^{(2)}(M, \phi)$, and similarly for $M^{\prime}$. So it suffices to show that $\rho^{(2)}(M, \phi)=\rho^{(2)}\left(M^{\prime}, \phi^{\prime}\right)$.

By the following lemma, $\operatorname{Ker}\{G \rightarrow \Gamma\}=\pi_{1}(W)^{(0)} / \pi_{1}(W)^{(n)}$ is amenable and in $D\left(\mathbb{Z}_{p}\right)$. From the amenability of $\operatorname{Ker}\{G \rightarrow \Gamma\}$ and $\Gamma$, the amenability of $G$ follows. Therefore by applying Theorem 7.1, we obtain $\rho^{(2)}(M, \phi)=\rho^{(2)}\left(M^{\prime}, \phi^{\prime}\right)$.

It can be seen that a similar argument works for $R=\mathbb{Q}$ and $\mathbb{Z}_{p}$.

Lemma 7.3. Suppose $\pi$ is a group over $\Gamma$.

(1) Let $R$ be either $\mathbb{Z}_{p}$ or $\mathbb{Z}_{(p)}$ and $\pi^{(n)}$ be the $R$-coefficient Vogel-Cohn local derived series of $\pi$ over $\Gamma$. Then $\pi^{(0)} / \pi^{(n)}$ is amenable and in $D\left(\mathbb{Z}_{p}\right)$ for any $n<\infty$.

(2) Let $\pi^{(n)}$ be the rational Vogel-Cohn local derived series of $\pi$ over $\Gamma$ and $R$ be any commutative ring. Then $\pi^{(0)} / \pi^{(n)}$ is amenable and in $D(R)$ for any $n<\infty$. 
Proof. For (1), consider the normal series $\left\{\pi^{(i)} / \pi^{(n)}\right\}_{0 \leq i \leq n}$ of $\pi^{(0)} / \pi^{(n)}$. The $i$-th quotient is isomorphic to $\pi^{(i)} / \pi^{(i+1)}$, which is the image of a map factoring through $H_{1}\left(\pi ; R\left[\pi / \pi^{(i)}\right]\right)$ by the definition of $\pi^{(i+1)}$. Since $H_{1}\left(\pi ; R\left[\pi / \pi^{(i)}\right]\right)$ has no torsion coprime to $p$ for both $R=\mathbb{Z}_{p}$ and $\mathbb{Z}_{(p)}$, so does $\pi^{(i)} / \pi^{(i+1)}$. It follows that $\pi^{(0)} / \pi^{(n)}$ is amenable and in $D\left(\mathbb{Z}_{p}\right)$ by Lemma 6.8. The second part is proved similarly.

\section{Applications}

8.1. Distinct homology cobordism types with the same simple homotopy type. In 8, Chang and Weinberger proved the following result on homeomorphism types of manifolds having the same simple homotopy type using $L^{2}$-signature invariants:

Theorem 8.1 (Chang-Weinberger [8]). Suppose $M$ is a closed $(4 k-1)$-manifold with $\pi=\pi_{1}(M), k \geq 2$. If $\pi$ has a nontrivial torsion element, then there exist infinitely many closed $(4 k-1)$-manifolds $M_{0}=M, M_{1}, M_{2}, \ldots$ such that each $M_{i}$ is simple homotopy equivalent and tangentially equivalent to $M$ but $M_{i}$ and $M_{j}$ are not homeomorphic for any $i \neq j$.

Using our results, we will prove a homology cobordism version of the ChangWeinberger theorem.

Theorem 8.2. Suppose $M$ is a closed $(4 k-1)$-manifold with $\pi=\pi_{1}(M), k \geq 2$. Let $p$ be prime and $\pi^{(n)}$ be the $\mathbb{Z}_{p}$ or $\mathbb{Z}_{(p)}$-coefficient Vogel-Cohn local derived series of $\pi$. If $\pi$ has a torsion element which remains nontrivial in $\pi / \pi^{(n)}$ for some $n$, then there exist infinitely many closed $(4 k-1)$-manifolds $M_{0}=M, M_{1}, M_{2}, \ldots$ such that each $M_{i}$ is simple homotopy equivalent and tangentially equivalent to $M$ but $M_{i}$ and $M_{j}$ are not homology cobordant for any $i \neq j$.

Note that $\pi / \pi^{(n)}$ has no torsion coprime to $p$ but may have $p$-torsion elements. We also remark that the $R \Gamma$-homology generalizations of Theorem 8.2 are true for $R=\mathbb{Z}, \mathbb{Z}_{p}$, and $\mathbb{Z}_{(p)}$ when $M$ is over an amenable group $\Gamma$. (Indeed the same proof works.)

In the $\mathbb{Z}_{p}$-coefficient case of Theorem 8.2. $\pi^{n}=\pi^{(n)}$ by Theorem 4.2. Therefore Theorem 8.2 specializes to the following result, which can also be proved directly by using the homology cobordism invariance of $L^{2}$-signatures associated to finite p-groups:

Corollary 8.3. Suppose $M$ is a closed $(4 k-1)$-manifold, $k>2$, and let $\left\{\pi^{n}\right\}$ be the $\mathbb{Z}_{p}$-derived series of $\pi=\pi_{1}(M)$. If $\pi$ has a torsion element which is not contained in $\pi^{n}$ for some $n$, then the conclusion of Theorem 8.2 holds.

Proof of Theorem 8.2. Following the argument of the proof of [8, Theorem 1], we consider the action of the surgery obstruction group $L_{4 k}(\pi)$ on the structure set $\mathcal{S}(M)$ of simple homotopy equivalences of closed $(4 k-1)$-manifolds into $M$ : for each $a \in L_{4 k}(\pi)$, by the Wall realization theorem there is a $4 k$-dimensional bordism, say $W_{a}$, between $M$ and another element $\mathcal{S}(M)$, say (represented by) $M_{a}$, over $\pi$ such that $a$ is represented by the $\mathbb{Z} \pi$-coefficient intersection form of $W_{a}$. We will show that we can choose infinitely many $a$, including $a=0$ (i.e., $M=M_{a}$ for some a) so that $M_{a}$ is not homology cobordant to $M_{a^{\prime}}$ for any $a \neq a^{\prime}$.

Let $G=\pi / \pi^{(n)}$ and $\phi: \pi_{1}(M) \rightarrow G$ and $\phi_{a}: \pi_{1}\left(M_{a}\right) \rightarrow G$ be the obvious maps. Then $\rho^{(2)}(M, \phi)-\rho^{(2)}\left(M_{a}, \phi_{a}\right)$ is equal to the $L^{2}$-signature defect of the intersection form on $H_{2 k}\left(W_{a} ; \mathcal{N} G\right)$. The proof of [8, Theorem 1] shows the following: for any group $G$ which is not torsion free, there exist infinitely many $o \in L_{4 k}(G)$ such that the $L^{2}$-signature defects of the forms representing $o$ are all nonzero and distinct. The elements $o$ are constructed as follows. Switching to the $\mathbb{Q}$-coefficients and 
considering forms over projective modules makes no difference, since the map of $L_{4 k}(G)=L_{4 k}(\mathbb{Z} G)$ into the relevant $L$-group $L_{4 k}(\mathbb{Q} G)$ induces an isomorphism modulo 8-torsion for any $G$. (We omit decorations in the $L$-group notation.) An order $r>1$ element in $G$ determines a map $L_{4 k}\left(\mathbb{Q}\left[\mathbb{Z}_{r}\right]\right) \rightarrow L_{4 k}(\mathbb{Q} G)$, and the images of direct sums of the $1 \times 1$ form $[1]$ on the projective $\mathbb{Q}\left[\mathbb{Z}_{r}\right]$-module $\mathbb{Q}$ has the desired property.

Returning to our case, we have an element in $\pi$ with finite order, say $d$, whose image in $G$ has order $r$ for some divisor $r>1$ of $d$. This gives us the followiung commutative diagram:

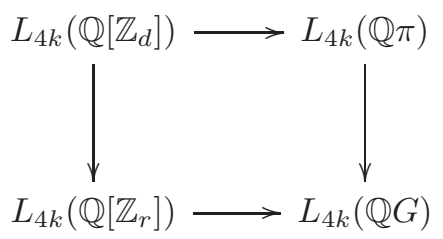

Since the class of the form $[1]$ in $L_{4 k}\left(\mathbb{Q}\left[\mathbb{Z}_{r}\right]\right)$ is in the image of $L_{4 k}\left(\mathbb{Q}\left[\mathbb{Z}_{d}\right]\right)$, the elements $o \in L_{4 k}(\mathbb{Q} G)$ described above are all in the image of $L_{4 k}(\mathbb{Q} \pi)$.

It follows that there are infinitely many $a \in L_{4 k}(\pi)$ such that $\rho^{(2)}(M, \phi)-$ $\rho^{(2)}\left(M_{a}, \phi_{a}\right)$ are all nonzero and distinct. From the homology cobordism invariance of $\rho^{(2)}(M, \phi)$ (see Theorem 7.2 ) the desired conclusion follows.

8.2. Homology cobordism types of 3-manifolds. In the below statement, $\pi^{(n)}$ denotes the $R$-coefficient Vogel-Cohn local derived series of a group $\pi$, where $R=$ $\mathbb{Z}_{p}, \mathbb{Z}_{(p)}$, or $\mathbb{Q}$.

Theorem 8.4. Suppose $M$ is a closed 3-manifold with $\pi=\pi_{1}(M)$. If $\pi^{(n)} / \pi^{(n+1)}$ is nontrivial for some $n>0$, then there exist infinitely many closed 3-manifolds $M_{0}=M, M_{1}, M_{2}, \ldots$ satisfying the following properties:

(1) For any $i$, there is a map $M \rightarrow M_{i}$ which induces isomorphisms on $H_{*}(-; \mathbb{Z})$ and on $\pi_{1}(-) / \pi_{1}(-)^{(k)}$ for any $k$.

(2) For any $i$ and $j, M_{i}$ and $M_{j}$ have identical Wall multisignatures (or equivalently Atiyah-Singer G-signatures).

(3) For any $i \neq j, M_{i}$ and $M_{j}$ are not homology cobordant.

Corollary 8.5. For the spherical 3-space form $M=S^{3} / Q_{8 n}$ with fundamental group $Q_{8 n}=\left\langle x, y \mid y^{2 n}=x^{2}=(x y)^{2}\right\rangle$ (see Milnor [25]), there are infinitely many closed 3-manifolds $M_{0}=M, M_{1}, M_{2}, \ldots$ such that the $M_{i}$ are homology equivalent to $M$ and have identical Wall multisignatures (or equivalently Atiyah-Singer $G$ signatures) and Harvey $L^{2}$-signature invariants $\rho_{n}$ [19], but no two of the $M_{i}$ are homology cobordant.

Proof. Due to our previous computation (Example 5.5),$\pi=Q_{8 n}$ has non-trivial $\pi^{(1)} / \pi^{(2)}$, where $\pi^{(n)}$ denotes the $\mathbb{Z}_{(2)}$-coefficient Vogel-Cohn local derived series. Therefore Theorem 8.4 applies. Harvey's invariant $\rho_{n}$ vanishes for any rational homology sphere.

\section{Remark 8.6.}

(1) While Harvey's $L^{2}$-invariants $\rho_{n}$ cannot distinguish the homology cobordism types in Corollary 8.5, our proof illustrates that $L^{2}$-signatures, combined with our injectivity theorem, are effective even for rational homology spheres.

(2) Our computation for $\pi=Q_{8 n}$ says that $\pi^{(n)} / \pi^{(n+1)}$ is a 2-group for $n \leq 1$; it enables an alternative proof of Corollary 8.5 using either the result of 
Levine 23], or the $L$-group-valued Hirzebruch-type invariants from iterated 2-covers which were defined and studied by the first author [4, 5].

Proof of Theorem 8.4. Choose a simple closed curve $\eta$ in $M$ representing a nontrivial element in $\pi^{(n)} / \pi^{(n+1)}$. We will use a construction sometimes called an infection along $\eta$ by a knot: identify a tubular neighborhood of $\eta$ with $\eta \times D^{2}$, choose a knot $K$, and let $E_{K}$ be the exterior of $K$. Let

$$
M(\eta, K)=\left(M-\left(\eta \times \operatorname{int} D^{2}\right)\right) \cup_{\partial} E_{K}
$$

where the pasting map is an orientation reversing homeomorphism on the boundary such that $\eta \times\{*\},\{*\} \times S^{1} \subset \eta \times S^{1}$ are identified with a meridian and longitude of $K$ on $\partial E_{K}$. We will show that the 3 -manifolds $M_{i}$ can be obtained in this way by choosing the knots $K$ appropriately.

Let $K_{0}$ be the unknot. Then there is a homology equivalence $E_{K} \rightarrow E_{K_{0}}$ that extends a homeomorphism on the boundary preserving the periphral structure. Pasting this with the identity map on $M-\left(\eta \times \operatorname{int} D^{2}\right)$, we obtain a homology equivalence $M(\eta, K) \rightarrow M\left(\eta, K_{0}\right)=M$.

Let $G=\pi_{1}(M(\eta, K))$. Then the induced map $G \rightarrow \pi$ is 2-connected on homology (for any $R$ ). Therefore by the injectivity theorem 3.8, the induced map $G / G^{(k)} \rightarrow$ $\pi / \pi^{(k)}$ is injective for any $k$. Since $\pi_{1}\left(E_{K}\right) \rightarrow \pi_{1}\left(E_{K_{0}}\right)$ is surjective, $G \rightarrow \pi$ is surjective. It follows that $G / G^{(k)} \rightarrow \pi / \pi^{(k)}$ is an isomorphism for any $k$.

Since $n \geq 1,[\eta]=0$ in $H_{1}(M)$. From this it follows that Wall's multisignatures of $M(\eta, K)$ are identical with those of $M$ (e.g., see [18] and Section 5 of [4]).

In order to distinguish the homology cobordism classes of the $M(\eta, K)$, we will use $L^{2}$-signatures. Consider $\rho^{(2)}(M(\eta, K), \psi)$, where $\psi$ is the quotient map $G \rightarrow$ $G / G^{(n+1)} \cong \pi / \pi^{(n+1)}$.

By previously known arguments (e.g., see [15], 13]), we have

$$
\rho^{(2)}(M(\eta, K), \psi)=\rho^{(2)}(M, \phi)+\rho^{(2)}\left(M_{K}, \alpha\right)
$$

where $\phi$ is the quotient map $\pi \rightarrow \pi / \pi^{(n+1)}, M_{K}$ is the zero-surgery manifold of $K$, and $\alpha: \pi_{1}\left(M_{K}\right) \rightarrow \pi / \pi^{(n+1)}$ is the map induced by the restriction of $\psi$ on $E_{K}$.

Let $d$ be the order of $[\eta]$ in $\pi / \pi^{(n+1)}$. ( $d$ may be $\infty$.) Since $\pi^{(n)}$ is a normal subgroup and $\pi_{1}\left(M_{K}\right)$ is normally generated by a meridian of $K$ which is identified with a parallel copy of $\eta$, Im $\alpha$ is contained in $\pi^{(n)} / \pi^{(n+1)}$ which is an abelian group. It follows that $\operatorname{Im} \alpha$ is isomorphic to $\mathbb{Z}_{d}$ (where $\mathbb{Z}_{\infty}$ is understood as the infinite cyclic group if $d=\infty)$. Therefore by the induction property, $\rho^{(2)}\left(M_{K}, \alpha\right)=\rho^{(2)}\left(M_{K}, \beta\right)$ where $\beta$ is the surjection $\pi_{1}\left(M_{K}\right) \rightarrow \operatorname{Im} \alpha \cong \mathbb{Z}_{d}$. Appealing to Lemma 8.7 below, we can choose infinitely many knots $K_{0}$ (=unknot), $K_{1}, K_{2}, \ldots$ such that the values $\rho^{(2)}\left(M_{K_{i}}, \beta\right)$ are mutually distinct. It follows that the $\rho^{(2)}\left(M\left(K_{i}, \eta\right), \phi_{K_{i}}\right)$ are all distinct. By the homology cobordism invariance of $\rho^{(2)}\left(M\left(K_{i}, \eta\right), \psi\right)$ (Theorem (7.2), the 3-manifolds $M_{i}=M\left(\eta, K_{i}\right)$ are not homology cobordant.

Lemma 8.7. Suppose $K$ is a knot in $S^{3}$ such that zero-surgery on $K$ yields the three manifold $M$. Let $A$ be a Seifert matrix for $K$. Let

$$
\sigma_{K}(\omega)=\operatorname{sign}\left((1-\omega) A+\left(1-\omega^{-1}\right) A^{T}\right)
$$

be the Levine-Tristram signature function of $K$ which is defined for $\omega \in S^{1} \subset \mathbb{C}$.

(1) [15, Proposition 5.1] For the abelianization map $\phi: \pi_{1}(M) \rightarrow \mathbb{Z}$, we have

$$
\rho^{(2)}(M, \phi)=\int_{S^{1}} \sigma_{K}(\omega) d \omega
$$

where the integral is over $S^{1}$ normalized to unit length. 
(2) Suppose $\phi_{d}: \pi_{1}(M) \rightarrow \mathbb{Z}_{d}$ is a surjection. Then

$$
\rho^{(2)}\left(M, \phi_{d}\right)=\frac{1}{d} \cdot \sum_{k=0}^{d-1} \sigma_{K}\left(\zeta_{d}^{k}\right)
$$

where $\zeta_{d}=e^{2 \pi \sqrt{-1} / d}$ is the dth primitive root of unity.

Proof. Construct in the usual way, a compact 4-manifold $W$ with boundary $M$ over $\mathbb{Z}$ whose $\mathbb{Z}\left[t, t^{-1}\right]$-coefficient intersection form is $(1-t) A+\left(1-t^{-1}\right) A^{T}$. (1) is proved in 15$]$ by computing the $L^{2}$-signature of the intersection form of $W$. One can prove (2) similarly, as follows: let $G=\mathbb{Z}_{d}$. Since $G$ is finite, the von Neumann group ring $\mathcal{N} G$ is equal to the ordinary group $\operatorname{ring} \mathbb{C} G$, and $d \cdot \operatorname{dim}^{(2)} M=\operatorname{dim}_{\mathbb{C}} M$ for any $\mathcal{N} G$-module $M$. Viewing the bordism $W$ described above as a bordism over $G$, the $\mathcal{N} G$-coefficient intersection form is exactly

$$
(1-g) A+\left(1-g^{-1}\right) A^{T}
$$

where $g$ is the generator of $G$ which is the image of the positive meridian. It can also be seen that the ordinary signature of $W$ is equal to $\sigma_{K}(1)$, which is always zero. From these observations (2) follows.

\section{Appendix A. REMARKS ON MOdUle AND RING LOCALIZATIONS}

A.1. Two definitions of the Bousfield module localization. In [2, Bousfield defined and studied localization of $Z \pi$-modules with respect to the class $H \mathbb{Z}$ of $Z \pi$-module homomorphisms $\alpha: A \rightarrow B$ such that the induced map $H_{i}(\pi ; A) \rightarrow$ $H_{i}(\pi ; B)$ is an isomorphism for $i=0$ and a surjection for $i=1$. His arguments readily extend to the case of a group $\pi$ over another group $\Gamma$. Namely, there is a localization functor with respect to the class, which will be denoted by $H \mathbb{Z}$ as well, of $R \pi$-module homomorphisms $\alpha: A \rightarrow B$ that induces an isomorphism $A \otimes_{R \pi} R \Gamma \rightarrow B \otimes_{R \pi} R \Gamma$ and a surjection $\operatorname{Tor}_{1}^{R \pi}(R \Gamma, A) \rightarrow \operatorname{Tor}_{1}^{R \pi}(R \Gamma, B)$.

Our goal is to show that the Bousfield localization with respct to $H \mathbb{Z}$ is equal to the localization with respect to the following class: let $\mathcal{W}$ be the collection of $R \pi$-module homomorphisms $\alpha: F \rightarrow F^{\prime}$ such that $F$ and $F^{\prime}$ are $R \pi$-free and $\alpha \otimes 1: F \otimes_{R \pi} R \Gamma \rightarrow F^{\prime} \otimes_{R \pi} R \Gamma$ is an isomorphism. Although surely well-known, we could find no proof in the literature for the following, and provide the proof here.

Theorem A.1. A R R-module $M$ is local with respect to $H \mathbb{Z}$ if and only if $M$ is local with respect to $\mathcal{W}$.

The following is an immediate consequence:

Corollary A.2. The localization with respect to $H \mathbb{Z}$ is equal to the localization with respect to $\mathcal{W}$.

Proof of Theorem. Observe that the class $\mathcal{W}$ is contained in $H \mathbb{Z}$. It follows that if $M$ is local with respect to $H \mathbb{Z}$, then $M$ is local with respect to $\mathcal{W}$.

To prove the converse, suppose $M$ is local with respect to $\mathcal{W}$, and consider a diagram

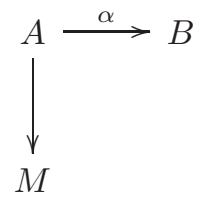

with $\alpha$ in $H \mathbb{Z}$. We have to show that there is a unique map $B \rightarrow M$ making the diagram commute. 
Let $N=\operatorname{Ker}\{\pi \rightarrow \Gamma\}$ and $I=\operatorname{Ker}\{R \pi \rightarrow R \Gamma\}$. Choose a set of generators $\left\{b_{i}\right\}_{i}$ of $B$. Since

$$
A \underset{R \pi}{\otimes} R \Gamma \cong B \underset{R \pi}{\otimes} R \Gamma \cong B /\langle g b-b \mid g \in N, b \in B\rangle
$$

we can write each $b_{i}$ as

$$
b_{i}=\alpha\left(a_{i}\right)+\sum_{j} r_{i j} b_{j}
$$

where $a_{i} \in A$ and $r_{i j} \in I$. We consider the following system of equations with variable $x_{i}$ (one variable for each generator $b_{i}$ ):

$$
S=\left\{x_{i}=a_{i}+\sum_{j} r_{i j} x_{j}\right\}_{i}
$$

When $S$ is a system of equation in $A$ as above, we define $A_{S}$ to be the module obtained from $A$ by adjoining the variables $x_{i}$ as additional generators satisfying the relations given by the equations in $S$. Precisely,

$$
A_{S}=\left(A \oplus F\left\langle x_{i}\right\rangle\right) /\left\langle x_{i}-a_{i}-\sum_{j} r_{i j} x_{j}\right\rangle
$$

where $F\left\langle x_{i}\right\rangle$ denotes the free $R \pi$-module generated by the $x_{i}$. Note that the natural map $A \rightarrow A_{S}$ induces an isomorphism $A \otimes_{R \pi} R \Gamma \rightarrow A_{S} \otimes_{R \pi} R \Gamma$ provided $r_{i j} \in I$. Also, in our case, there is a well-defined map $A_{S} \rightarrow B$ sending $x_{i}$ to $b_{i}$.

Choose a surjection $F \rightarrow A$ of a free $R \pi$-module $F$. Let $S^{\prime}$ be a system of equations in $F$ which is a lift of $S$, that is, $S^{\prime}$ is obtained from $S$ by replacing each $a_{i}$ by a pre-image of $a_{i}$ in $F$. We have the following commutative diagram:

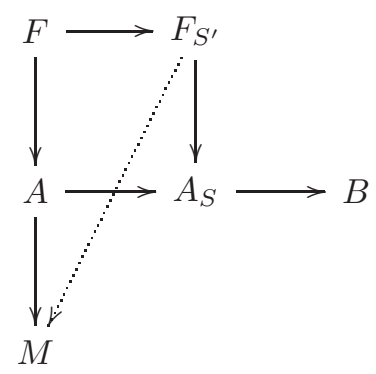

Since $F \rightarrow F_{S^{\prime}}$ is in $\mathcal{W}$, there is $F_{S^{\prime}} \rightarrow M$ making the diagram commute.

It can be checked that $\operatorname{Ker}\left\{F_{S^{\prime}} \rightarrow A_{S}\right\}$ is equal to the image of $\operatorname{Ker} F \rightarrow A$ under $F \rightarrow F_{S^{\prime}}$. Therefore, $F_{S^{\prime}} \rightarrow M$ induces $A_{S} \rightarrow M$. Observe the following facts:

(1) $A_{S} \otimes_{R \pi} R \Gamma \cong A \otimes_{R \pi} R \Gamma \cong B \otimes_{R \pi} R \Gamma$.

(2) $\operatorname{Tor}_{1}^{R \pi}\left(A_{S}, R \Gamma\right) \rightarrow \operatorname{Tor}_{1}^{R \pi}(B, R \Gamma)$ is surjective, since so is $\operatorname{Tor}_{1}^{R \pi}(A, R \Gamma) \rightarrow$ $\operatorname{Tor}_{1}^{R \pi}(B, R \Gamma)$.

Let $K=\operatorname{Ker}\left\{A_{S} \rightarrow B\right\}$. Looking at the Tor long exact sequence obtained from $0 \rightarrow K \rightarrow A_{S} \rightarrow B \rightarrow 0$, from the above observations it follows that $R \Gamma \otimes_{R \pi} K=0$. By the lemma stated and proved below, it follows that the image of $K$ in $M$ under $A_{S} \rightarrow M$ is zero. Therefore, from (1), it follows that there is an induced map $B \rightarrow M$. The map $B \rightarrow M$ is uniquely determined since $F_{S^{\prime}} \rightarrow M$ is unique.

Definition A.3. For $\pi$ over $\Gamma$, an $R \pi$-module $N$ is perfect if $N \otimes_{R \pi} R \Gamma=0$.

Lemma A.4. If $M$ is local with respect to $\mathcal{W}$, then $M$ has no nontrivial perfect submodules. Consequently any map of a perfect module into $M$ is zero.

Proof. Suppose $N$ is a perfect submodule of $M$. Choose generators $\left\{a_{i}\right\}$ of $N$. Since $N \otimes_{R \pi} R \Gamma=N /\langle g a-a \mid g \in \operatorname{Ker}\{\pi \rightarrow \Gamma\}, a \in N\rangle=0$, we can write each 
$a_{i}$ as $a_{i}=\sum_{j} r_{i j} a_{j}$ where $r_{i j} \in I=\operatorname{Ker}\{R \pi \rightarrow R \Gamma\}$. Let $F\left\langle x_{i}\right\rangle$ be the free $R \pi$ module generated by variables $x_{i}$ (one variable $x_{i}$ for each $b_{i}$ ) as before, and define $\alpha: F\left\langle x_{i}\right\rangle \rightarrow F\left\langle x_{i}\right\rangle$ by $\alpha\left(x_{i}\right)=x_{i}-\sum_{j} r_{i j} x_{j}$. Consider

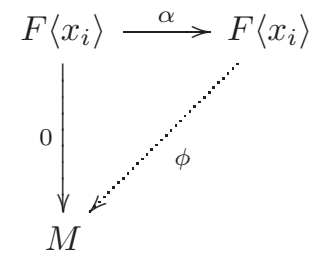

Observe that $\alpha$ is in $\mathcal{W}$. The map $\phi: F\left\langle x_{i}\right\rangle \rightarrow M$ given by $\phi\left(x_{i}\right)=a_{i}$ makes the diagram commute. Instead of $\phi$, the zero map also makes it commute. Therefore, since $M$ is local with respect to $\mathcal{W}, \phi=0$ by the uniqueness. It follows that $a_{i}=0$.

A.2. Ring localization as module localization. Suppose $\pi$ is a group, $R$ is a commutative ring with unity, $\Omega$ is a collection of $R \pi$-module homomorphisms, and $E$ is a localization functor on the category of $R \pi$-modules with respect to $\Omega$, which is endowed with a natural transformation $M \rightarrow E(M)$. Our goal is to give a proof that $E(\mathbb{Z} \pi)$ is a ring localization in the sense of Section 2.2

Theorem A.5. There is a ring structure on $\Lambda=E(R \pi)$ satisfying the following:

(1) The map $R \pi \rightarrow \Lambda$ is a ring homomorphism.

(2) For any $R \pi$-module $M$, there is a natural isomorphism $E(M) \cong M \otimes_{R \pi} \Lambda$.

(3) For any $\alpha: A \rightarrow B$ in $\Omega, \alpha \otimes 1: A \otimes_{R \pi} \Lambda \rightarrow B \otimes_{R \pi} \Lambda$ is an isomorphism.

(4) $R \pi \rightarrow \Lambda$ is initial among objects satisfying (3), that is, if a ring homomorphism $R \pi \rightarrow \Lambda^{\prime}$ satisfies (3), then there is a unique ring homomorphism $\Lambda \rightarrow \Lambda^{\prime}$ making the following diagram commute:

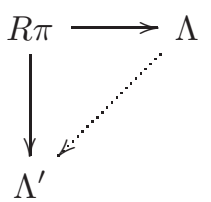

Proof. (1) Denote by $i$ the map $R \pi \rightarrow E(R \pi)=\Lambda$. The ring structure on $\Lambda$ is defined as follows. Since $R \pi \otimes_{R \pi} \Lambda \cong \Lambda$ and the functor $E$ commutes with direct sum, for any free $R \pi$-module $F$, we have the following commutative diagram:

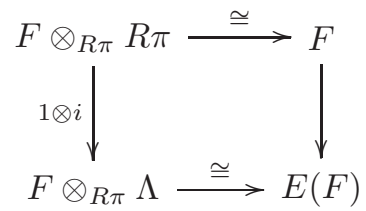

Choose a free resolution $F_{0} \rightarrow F_{1} \rightarrow \Lambda$ over $R \pi$. Then we have

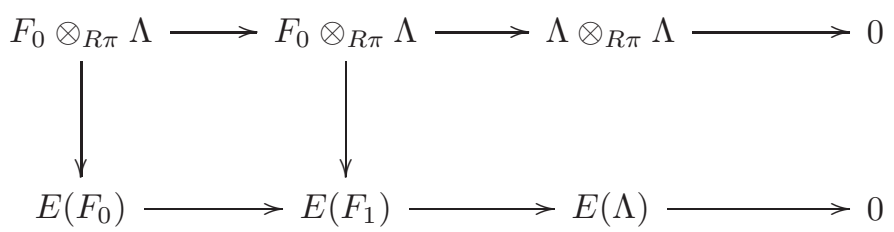

Rows are exact since tensoring and $E$ are right exact, and vertical arrows are isomorphisms. Since $E$ is an idempotent, it follows that there is an isomorphism

$$
m: \Lambda \underset{R \pi}{\otimes} \Lambda \longrightarrow \Lambda=E(\Lambda)
$$


which gives ring multiplication on $\Lambda$. Obviously $R \pi \rightarrow \Lambda$ is a ring homomorphism.

(2) For a given $R \pi$-module $M$, choose a presentation $F_{0} \rightarrow F_{1} \rightarrow M \rightarrow 0$ with $F_{0}, F_{1}$ free. Replacing the resolution of $\Lambda$ in the above argument of (1) by the resolution of $M$, we obtain an isomorphism $M \otimes_{R \pi} \Lambda \rightarrow E(M)$. It can be verified that this isomorphism is independent of the choice of the presentation.

(3) Any $\alpha: A \rightarrow B$ in $\Omega$ induces an isomorphism $E(A) \rightarrow E(B)$ since $E$ is a localization with respect to $\Omega$. Since $E(A) \cong A \otimes_{R \pi} \Lambda$, the desired conclusion follows.

(4) Suppose a ring homomorphism $j: R \pi \rightarrow \Lambda^{\prime}$ satisfies (3). First we will show that $\Lambda^{\prime}$ is a local $R \pi$-module with respect to $\Omega$. Suppose $\alpha: A \rightarrow B$ in $\Omega$ and $\phi: A \rightarrow \Lambda^{\prime}$ is given.

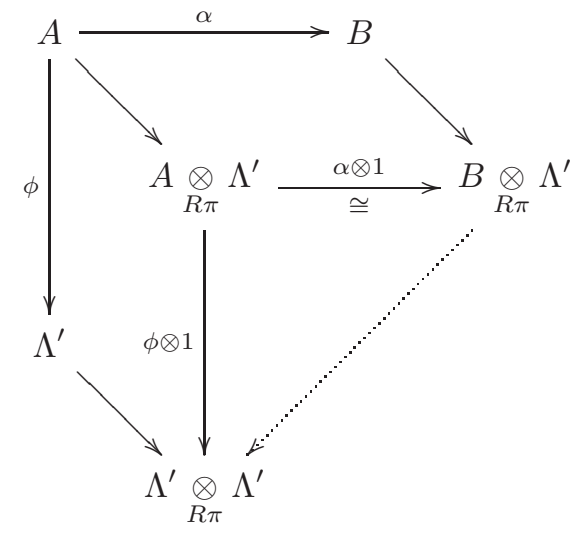

In the above diagram, $\alpha \otimes 1$ is an isomorphism by (2). Let $m^{\prime}: \Lambda^{\prime} \otimes_{R \pi} \Lambda^{\prime} \rightarrow \Lambda^{\prime}$ be the ring multiplication and let $\psi: B \rightarrow \Lambda^{\prime}$ be the composition of $B \rightarrow B \otimes_{R \pi} \Lambda^{\prime}$ and $m^{\prime}(\phi \times 1)(\alpha \otimes 1)^{-1}$. Then $\psi \alpha=\phi$. If $\psi^{\prime} \alpha=\phi$ for some $\psi^{\prime}: B \rightarrow \Lambda^{\prime}$, then $\psi^{\prime} \otimes 1: B \otimes_{R \pi} \Lambda^{\prime} \rightarrow \Lambda^{\prime} \otimes_{R \pi} \Lambda^{\prime}$ should be equal to $(\phi \otimes 1)(\alpha \otimes 1)^{-1}$, being the unique map making the triangle commute. From this it follows that $\phi^{\prime}=\phi$ by a diagram chase. This proves that the $R \pi$-module $\Lambda^{\prime}$ is local.

By the universal property of the module localization $\Lambda=E(R \pi)$, there is a unique $R \pi$-module homomorphism $f: \Lambda \rightarrow \Lambda^{\prime}$ making the following diagram commute:

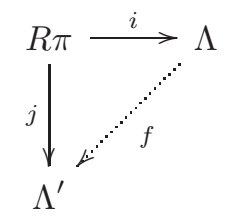

We claim that $f$ is a ring homomorphism. To prove this, consider the following diagram, where $m, m_{0}, m^{\prime}$ are ring multiplication.

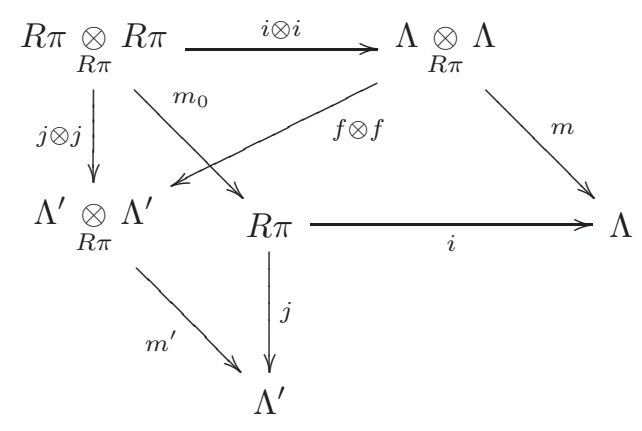


It can be seen that the diagram commutes. Now look at $m^{\prime}(f \otimes f) m^{-1}: \Lambda \rightarrow \Lambda^{\prime}$. Then by a diagram chase, one can verify

$$
m^{\prime}(f \otimes f) m^{-1} i=m^{\prime}(f \otimes f)(i \otimes i) m_{0}^{-1}=m^{\prime}(j \otimes j) m_{0}^{-1}=j .
$$

From the uniqueness of $f$, it follows that $m^{\prime}(f \otimes f) m^{-1}=f$, that is, $f$ is a ring homomorphism.

\section{REFERENCES}

[1] A. K. Bousfield, Homological localizations of spaces, groups, and $\pi$-modules, Localization in group theory and homotopy theory, and related topics (Sympos., Battelle Seattle Res. Center, Seattle, Wash., 1974), Springer, Berlin, 1974, pp. 22-30. Lecture Notes in Math., Vol. 418.

[2] . The localization of spaces with respect to homology, Topology 14 (1975), 133-150.

[3] S. E. Cappell and J. L. Shaneson, The codimension two placement problem and homology equivalent manifolds, Ann. of Math. (2) 99 (1974), 277-348.

[4] J. C. Cha, Link concordance, homology cobordism, and Hirzebruch-type defects from iterated p-covers, arXiv:0705.0088, to appear in J. Eur. Math. Soc.

[5] _ Structure of the string link concordance group and Hirzebruch-type invariants, arXiv:0709.2968, to appear in Indiana Univ. Math. J.

[6] _ Injectivity theorems and algebraic closures of groups with coefficients, Proc. London Math. Soc. 96 (2008), no. 1, 227-250.

[7] _ Topological minimal genus and $L^{2}$-signatures, Algebr. Geom. Topol. 8 (2008), 885909.

[8] S. Chang and S. Weinberger, On invariants of Hirzebruch and Cheeger-Gromov, Geom. Topol. 7 (2003), 311-319 (electronic).

[9] T. D. Cochran and S. Harvey, Homology and derived p-series of groups, arXiv:math.GT/0702894, to appear in J. London Math. Soc.

[10] _ Homology and derived series of groups, Geom. Topol. 9 (2005), 2159-2191 (electronic).

[11] _ Homological stability of series of groups, arXiv:0802.2390, 2008.

[12] - Homology and derived series of groups. II. Dwyer's theorem, Geom. Topol. 12 (2008), no. $1,199-232$.

[13] T. D. Cochran, S. Harvey, and C. Leidy, Knot concordance and higher-order Blanchfield duality, Geom. Topol. 13 (2009), no. 3, 1419-1482.

[14] T. D. Cochran, K. E. Orr, and P. Teichner, Knot concordance, Whitney towers and $L^{2}$ signatures, Ann. of Math. (2) 157 (2003), no. 2, 433-519.

[15] - Structure in the classical knot concordance group, Comment. Math. Helv. 79 (2004), no. $1,105-123$

[16] P. M. Cohn, Free rings and their relations, Academic Press, London, 1971, London Mathematical Society Monographs, No. 2.

[17] E. Dror Farjoun, K. Orr, and S. Shelah, Bousfield localization as an algebraic closure of groups, Israel J. Math. 66 (1989), no. 1-3, 143-153.

[18] S. Friedl, Link concordance, boundary link concordance and eta-invariants, Math. Proc. Cambridge Philos. Soc. 138 (2005), no. 3, 437-460.

[19] S. Harvey, Homology cobordism invariants and the Cochran-Orr-Teichner filtration of the link concordance group, Geom. Topol. 12 (2008), 387-430.

[20] P. Heck, Knot concordance in three manifolds, Ph.D. thesis, Indiana University, 2009.

[21] J.-Y. Le Dimet, Cobordisme d'enlacements de disques, Mém. Soc. Math. France (N.S.) (1988), no. 32 , ii +92 .

[22] J. P. Levine, Link concordance and algebraic closure. II, Invent. Math. 96 (1989), no. 3, 571-592.

[23] — Link invariants via the eta invariant, Comment. Math. Helv. 69 (1994), no. 1, $82-119$.

[24] W. Lück, $L^{2}$-invariants: theory and applications to geometry and $K$-theory, Ergebnisse der Mathematik und ihrer Grenzgebiete. 3. Folge. A Series of Modern Surveys in Mathematics [Results in Mathematics and Related Areas. 3rd Series. A Series of Modern Surveys in Mathematics], vol. 44, Springer-Verlag, Berlin, 2002.

[25] J. Milnor, Groups which act on $S^{n}$ without fixed points, Amer. J. Math. 79 (1957), 623-630.

[26] A. L. T. Paterson, Amenability, Mathematical Surveys and Monographs, vol. 29, American Mathematical Society, Providence, RI, 1988.

[27] J. Stallings, Homology and central series of groups, J. Algebra 2 (1965), 170-181. 
[28] R. Strebel, Homological methods applied to the derived series of groups, Comment. Math. Helv. 49 (1974), 302-332.

[29] P. Vogel, Localization of spaces with respect to a class of maps, Preprint, Univ. de Nantes, 1978.

[30] (1982), no. 55, 165-206.

Department of Mathematics and Pohang Mathematics Institute, Pohang University of Science and Technology, Pohang, Gyungbuk 790-784, Republic of Korea

E-mail address: jccha@postech.ac.kr

Department of Mathematics, Indiana University, Bloomington, Indiana 47405, USA

E-mail address: korr@indiana.edu 